Pacific Journal of Mathematics

A FOURIER TRANSFORM THEOREM ON NILMANIFOLDS 


\title{
A FOURIER TRANSFORM THEOREM ON NILMANIFOLDS AND NIL-THETA FUNCTIONS
}

\author{
RICHARD PENNEY
}

\begin{abstract}
A theorem describing the image of the Euclidean Fourier transform on certain nilmanifolds is proven. As an application, we compute the Fourier transform of a certain class of distributions on a manifold which is analogous with the class of classical theta functions on $C$. It is shown that our theta functions satisfy a functional equation which generalizes the functional equation satisfied by the Jacobi theta functions.
\end{abstract}

In this paper we present what we feel is a fascinating theorem concerning the Euclidian Fourier transform of certain functions on certain nilmanifolds. As an application of our Fourier transform theorem, we compute the Fourier transform of a certain class of distributions on a nilmanifold which is analogous to the class of theta functions on $\boldsymbol{C}$. It is shown that our theta distributions satisfy a functional equation which generalizes the functional equation satisfied by the Jacobi theta functions.

To describe our Fourier transform theorem, let $\mathscr{B}$ be an associative, finite dimensional, nilpotent algebra over $R$. $\mathscr{B}$ is said to be left commutative if $x y w=y x w$ for all $x, y, w \in \mathscr{B}$. $\mathscr{B}$ is said to be $H A T$ if $\mathscr{B}$ is left commutative and $\operatorname{dim} \mathscr{F}(\mathscr{B})=1$ $(\mathscr{Z}(\mathscr{B})=$ center of $\mathscr{B})$. In this case $\mathscr{Z}(\mathscr{B})$ annihilates $\mathscr{B}$ because the two sided annihilator of $\mathscr{B}$ is nontrivial and central. $\mathscr{L}(\mathscr{B})$ is, in fact, precisely the two sided annihilator of $\mathscr{B}$. $\mathscr{B}$ defines a nilpotent Lie algebra by setting $[x, y]=x y-y x$. The corresponding Lie group can be realized as the space $\mathscr{B}$ along with the product $x * y=x+y+x y$. The exponential map $\exp _{\mathscr{G}}$ is given by $\exp _{\mathscr{Q}} x=\sum_{n=1}^{\infty} x^{n} / n !$. The inverse of $x \in \mathscr{B}$ is $\bar{x}=\sum_{n=1}^{\infty}(-1)^{n} x^{n}$. (We choose the notation $\bar{x}$ in preference to $x^{-1}$ to avoid confusion with inverses in $\mathscr{B}$, which of course don't exist). When we wish to think of $\mathscr{B}$ as a group we will use the notation $\left(\mathscr{B},{ }^{*}\right)$.

Now suppose that $\mathscr{B}$ is a $H A T$ algebra which contains a closed subgroup $\Gamma$ such that the component of the identity of $\Gamma$ is $\mathscr{Z}(\mathscr{B})$ and $\Gamma \backslash\left(\mathscr{B},{ }^{*}\right)$ is compact. Let $\mu$ be an abelian character of $\Gamma$ which is nontrivial on $\mathscr{Z}(\mathscr{B})$. A function $\psi$ on $\mathscr{B}$ is said to be $\mu$-primary if $\psi$ is $C^{\infty}$ on $\mathscr{B}$ and $\psi$ satisfies $\psi(\gamma * x)=\mu(\gamma) \psi(x)$ for all $\gamma \in \Gamma$ and $x \in \mathscr{B}$. Our Fourier transform theorem describes the Fourier transform of the $\mu$-primary functions. 
The sense in which we mean the Fourier transform needs some explanation. Let $d \mathscr{B}$ denote an additive Haar measure on the vector space $\mathscr{B}$. Let $\mathscr{S}(\mathscr{B})$ denote the Schwartz space of rapidly decreasing functions on $\mathscr{B}$. If $\psi$ is any locally integrable function on $\mathscr{B}$ that grows at most polynomially, we may use $\psi$ to define a Schwartz distribution (also denoted $\psi$ ) on $\mathscr{S}(\mathscr{B})$ by setting

$$
\langle f, \psi\rangle=\int_{\mathscr{G}} f(x) \psi(x) d \mathscr{B}(x) .
$$

Schwartz distributions on $\mathscr{B}$ have Fourier transforms which are Schwartz distributions on $\mathscr{B}^{*}$. It is in this distributional sense that we take the word "Fourier transform". The specific conventions we adopt for the Fourier transform are described below for $f \in \mathscr{S}\left(\mathscr{B}^{*}\right)$. The Haar measure $d \mathscr{B}^{*}$ on $\mathscr{B}^{*}$ is normalized by requiring that $\mathscr{F}^{-1} \mathscr{F}=I$.

$$
\begin{array}{ll}
\mathscr{F} f(\lambda)=\int_{\mathscr{\mathscr { B }}} f(x) \exp -2 \pi i\langle x, \lambda\rangle d \mathscr{B}(x) & \left(\lambda \in \mathscr{B}^{*}\right) \\
\mathscr{F}^{-1} f(x)-\int_{\mathscr{F}^{*}} g(\lambda) \exp 2 \pi i\langle x, \lambda\rangle d \mathscr{B}^{*}(\lambda) & (x \in \mathscr{B}) .
\end{array}
$$

Now $\mathscr{B}$ anti-acts on $\mathscr{B}^{*}$ on both the left and the right according to the formulas

$$
\begin{aligned}
& x \cdot \lambda(y)=\lambda(x y) \\
& \lambda \cdot x(y)=\lambda(y x) .
\end{aligned}
$$

$(\mathscr{B}, *)$ also anti-acts on $\mathscr{B}^{*}$ by setting $x * \lambda=\lambda+x \lambda$ and $\lambda * x=\lambda+$ $\lambda x$. Each coset of $\mathscr{B}^{*} /(\mathscr{B}, *)$ and $\left(\mathscr{B},{ }^{*}\right) \backslash \mathscr{B}^{*}$ is an affine subspace of $\mathscr{B}^{*}$. Hence each such coset carries an essentially unique Haar measure. Our first theorem on the Fourier transform of $\mu$-primary functions is the following.

THEOREM A. Let $\psi$ be $\mu$-primary, then $\mathscr{F} \psi$ is supported in a countable union of $\mathscr{B}^{*} /(\mathscr{B}, *)$ cosets. On each of these cosets, $\mathscr{F}^{*} \psi$ is a $C^{\infty}$ function times the Haar measure of the given coset.

This theorem, however, is just the beginning of the story. We show that $\mathscr{F} \psi$ is supported in a finite union of $\Gamma \backslash \mathscr{B}^{*} /(\mathscr{B}, *)$ double cosets. Let $\lambda_{1}, \cdots, \lambda_{n}$ be a complete set of representatives for these double cosets. Let $\mathscr{C}\left(\lambda_{i}\right)=\lambda_{i} * \mathscr{B}$. From the transformation properties of $\psi$ under $\Gamma, \mathscr{F} \psi$ is determined by its restrictions $\psi_{i}$ to $\mathscr{M}\left(\lambda_{i}\right)$. On $\mathscr{C}\left(\lambda_{i}\right), \psi_{i}$ is a $C^{\infty}$ function. For $x \in \mathscr{B}$, let

$$
\widetilde{\psi}_{i}(x)=\exp 2 \pi i\left\langle\bar{x}, \lambda_{i}\right\rangle \psi_{i}\left(\lambda_{i} * \bar{x}\right) .
$$


We prove the following

THEOREM B. For each $i$ there is a closed subgroup $\Delta_{i}$ and a unique character $\chi_{i}$ of $\Delta_{i}$ such that

(1) $\widetilde{\psi}_{i}(\delta * x)=\chi_{i}(\delta) \widetilde{\psi}_{i}(x)$ for all $\delta \in \Delta$.

(2) $\widetilde{\psi}_{i}$ is $C^{\infty}$ on the connected hull $\mathscr{L}_{i}$ of $\Delta_{i}$ and $\psi_{i}$ is Schwartz transverse to $\mathscr{K}_{i}$.

(3) Let $\mathscr{C}_{i}$ denote the space of functions satisfying (2) above relative to $\Delta_{i}$. Then the mapping $\psi \rightarrow\left(\widetilde{\psi}_{1}, \cdots, \widetilde{\psi}_{n}\right)$ of into $\sum_{i} \oplus \mathscr{C}_{i}$ is an isomorphism of vector spaces.

(4) Let $R(x)$ denote right translation of functions by $x \in \mathscr{B}$ $(R(x) f(y)=f(y * x))$. Then $R(x)\left(\widetilde{\psi}_{i}\right)=(R(x) \tilde{\psi})_{i}$.

It is also true that the spaces in question (i.e., the spaces $\mathscr{C}_{i}$ above and the space of $\mu$-primary functions) can all be given unitary structures in a natural way and that, upon suitable normalization, the mapping $\psi \rightarrow\left(\psi_{1}, \cdots, \psi_{n}\right)$ becomes a unitary isomorphism. Theorems $\mathrm{A}$ and $\mathrm{B}$ taken together amount to an explicit computation of the Fourier transform of an arbitrary $\mu$-primary function $\psi$, especially in the case that the induced representation ind $(\Gamma,(\mathscr{B}, *), \mu)$ is irreducible. In this case there is only one space $\mathscr{C}_{1}$ and the mapping $\psi \rightarrow \widetilde{\psi}_{i}$ is an intertwining operator for two irreducible representations ind $(\Gamma,(\mathscr{B}, *), \mu)$ and ind $\left(\Delta_{1},(\mathscr{B}, *), \chi_{1}\right)$. The science of constructing such operators is well developed (see [4] e.g.). Typically $\psi_{1}$ will be expressible in the form

$$
\psi_{1}(x)=\int_{\Gamma_{0} \cap K \backslash K} \psi(k x) \bar{\chi}_{i}(k) d k
$$

where $K$ is an abelian normal subgroup of $\Delta_{i}$ and $A_{i}$ is trivial on the co-compact subgroup $\Gamma_{0} \cap K$ of $K$. A remarkable aspect of such formulas in that $\mathscr{F} \psi(\lambda)$ for a given $\lambda$ depends only on the values of $\psi$ along certain cosets of certain relatively small subgroups of $(\mathscr{B}, *)$.

As an application we consider the following situation. Let $\mathscr{S}$ be an abelian, nilpotent, finite dimensional algebra over $R$. Let $B$ be a symmetric, bi-linear form on $\mathscr{S}$ which satisfies $B(x y, w)=$ $B(y, x w)$. We define the scalar $\log$ function $l$ on $\mathscr{S}$ by

$$
l(x)_{.}=\sum_{n=2}^{\infty}(-1)^{n+1} B\left(x, x^{n-1}\right) / n .
$$

Note that the sum is really finite so $l$ is a polynomial.

We suppose that there is a vector lattice $\Lambda \subset \mathscr{S}$ such that $\Lambda^{2} \subset$ $\Lambda$ and $B(\Lambda, \Lambda) \subset Z$. Let $\sigma$ be a complex number. We let $\theta_{\sigma}(x)$ denote the following (usually divergent) series on $\mathscr{S}$. 


$$
\theta_{\sigma}(x)=\sum_{n \in A} \exp 2 \pi i(\sigma l(n)+B(n, x)) .
$$

This series is called the theta series of period $\sigma$ associated with $\mathscr{S}$. If $\sigma$ is real each term of $\theta_{\sigma}$ is bounded so $\theta_{\sigma}$ may be considered as a sequence of Schwartz distributions on $\mathscr{S}$. We shall show that in this case $\theta_{\sigma}$ converges in the sense of distribution theory. One other case in which convergence occurs is where $\mathscr{S}$ is $\boldsymbol{R}^{n}$ with trivial multiplication and $B$ is the usual scalar product. In this case $l(x)=-\|x\|^{2} / 2$. Let $\Lambda=Z^{n}$. Then

$$
\theta_{\sigma}(x)=\sum \exp 2 \pi i\left(-\sigma\|n\|^{2} / 2+n \cdot x\right) .
$$

If $\sigma$ has real imaginary part, this sum converges uniformly in $x$ and $y$. The limit is a slight variant of the classical Jacobi theta on $\boldsymbol{C}^{n}$.

The $\theta_{\sigma}$ series behave in many ways like classical theta functions. For example, if $\sigma$ is real we prove the following theorem (which does not use the Fourier transform result).

THEOREM C. If $\sigma$ is real, then as a distribution

$$
\theta_{\sigma}(x)=K(\sigma) \sum_{n \in A} \exp 2 \pi i \sigma l\left(\sigma^{-1}(x+n)\right)
$$

where $K(\sigma)$ is some constant.

In the case that $\mathscr{S}=\boldsymbol{R}^{2}$ with trivial multiplication and $B$ is the usual scalar product, this result is equivalent with the usual transformation identity of the Jacobi theta functions under the modular group. We also outline a technique for computing $K(\sigma)$.

To apply the Fourier transform theorem we consider a slightly larger class of functions-the mollified theta functions. A linear mapping $\tau_{0}: \mathscr{S} \rightarrow \mathscr{S}$ is said to be a mollifier if $\tau_{0}$ satisfies $\tau_{0}(x y)=$ $x \tau_{0} y=0$ for all $x, y \in \mathscr{S}$ and $B\left(x, \tau_{0} y\right)=B\left(\tau_{0} x, y\right)$. The 0 map is a mollifier, although $\mathscr{S}$ always has nontrivial mollifiers too. For example, if $e \in \mathscr{S}$ and $e \mathscr{S}^{2}=0$ then the mapping $\tau_{0}(x)=e x$ is a mollifier. The two variable series

$$
\theta_{\sigma, \tau_{0}}(x, y)=\left[\exp 2 \pi i\left(\sigma l(x)+B\left(\tau_{0} x, y\right)\right)\right] \theta_{\sigma}(y-\sigma x)
$$

is called the mollified theta series with period $\sigma$ and mollifier $\tau_{0}$. Using our Fourier transform theorem we may compute the Fourier transfer on $\mathscr{S} \times \mathscr{S}$ of any mollified theta functions. See $\S$ III.

II. General theory. We begin with a proof of Theorem A above. Throughout this work we shall write distributions in functional notation. For example if $\psi$ is a Schwartz distribution on a 
vector space $V$ and $A$ is a topological isomorphism of $V$ into $V$ which preserves the Haar measure $d V$, and preserves $\mathscr{S}(V)$, then by $x \rightarrow \psi(A(x))$ we shall mean the distribution $\psi_{A}$ defined by $\left\langle f, \psi_{A}\right\rangle=$ $\left\langle f \circ A^{-1}, \psi\right\rangle$. Similarly, if $g(x)$ is a $C^{\infty}$ function which has bounded derivatives of all orders, by $x \rightarrow g(x) \psi(x)$ we shall mean the distribution $f \rightarrow\langle f g, \psi\rangle$.

We consider $\mu$-primary functions as distributions on $\mathscr{B}$ as usual and let $\mathscr{F}$ denote the Fourier transform as described above. First we shall prove the statements about the support of $\mathscr{F} \psi$.

LEMMA 1. Let $\psi$ be a Schwartz distribution on $\mathscr{B}$. Let $L(g) \psi$ be the distribution $x \rightarrow \psi(\bar{g} * x)$ and let $R(g) \psi$ be $x \rightarrow \psi(x * g)$. (See above comments for notation). Then

$$
\begin{aligned}
& \mathscr{F}(L(g) \psi)(\lambda)=\exp -2 \pi i\langle g, \lambda\rangle \mathscr{F} \psi(g * \lambda) \\
& \mathscr{F}(R(g) \psi)(\lambda)=\exp -2 \pi i\langle\bar{g}, \lambda\rangle \mathscr{F} \psi(\lambda * \bar{g}) .
\end{aligned}
$$

Proof. Straightforward.

Now let $\mathscr{L}(\mathscr{B})$ be the left annihilator of $\mathscr{B}$,

$$
\mathscr{L}(\mathscr{B})=\{x \in \mathscr{B} \mid x \mathscr{B}=\{0\}\} .
$$

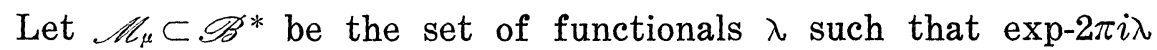
equals $\mu$ on $\Gamma \cap \mathscr{L}(\mathscr{B})=\Gamma_{\mathscr{L}}$. It can easily be seen that $\mathscr{M}_{\mu}$ is nonempty, although the nontriviality also follows from the corollary below.

Lemma 2. Let $\psi$ be $\mu$-primary. Then $\mathscr{F} \psi$ has a restriction to $\mathscr{L}_{\mu}$ in the sense that if $f=g$ on $\mathscr{M}_{\mu}$, then $\mathscr{F} \psi(f)=\mathscr{F} \psi(g)$ for $f, g \in \mathscr{S}\left(\mathscr{B}^{*}\right)$.

Proof. There is a character $\tilde{\mu}$ of the abelian group $\mathscr{L}(\mathscr{B})$ which equals $\mu$ on $\Gamma_{\mathscr{S}}$. There is a functional $\lambda_{0} \in \mathscr{C}_{\mu}$ such that $\exp 2 \pi i \lambda_{0} \mid \mathscr{L}(\mathscr{B})=\tilde{\mu}$. Let $\psi$ be $\mu$-primary and let

$$
\mathscr{N}(x)=\exp -2 \pi i\left\langle x, \lambda_{0}\right\rangle \psi(x),
$$

Then $\mathscr{N}(\gamma x)=\mathscr{N}(x)$ for $\gamma \in \Gamma_{\mathscr{L}}$ and $x \in \mathscr{B}$. Also

$$
\mathscr{F} \psi(\lambda)=\mathscr{F} \mathscr{N}\left(\lambda-\lambda_{0}\right)
$$

so it suffices to show that if $f=0$ on $\mathscr{H}_{\mu}-\lambda_{0}$, then $\mathscr{N}(f)=0$. To see this, let $g=\mathscr{F}^{-1} f, g \in \mathscr{S}(\mathscr{B})$. Let

$$
g_{0}(x)=\sum_{\mathscr{L}} g(x+\gamma) \text {. }
$$


This converges to a $C^{\infty}$ function because $g$ is Schwartz. It follows from reasoning similar to that found in the proof of Corollary 19 below that $g_{0}=0$ implies that $\mathscr{N}(g)=0$. But, from the Poisson summation formula

$$
g_{0}(x)=\int_{\left(\Gamma_{\mathscr{E}}\right)^{*}} f(\lambda) \exp -2 \pi i\langle x, \lambda\rangle d \lambda
$$

where $\left(\Gamma_{\mathscr{E}}\right)^{*}=\left\{\lambda \in \mathscr{B}^{*} \mid \lambda\left(\Gamma_{\mathscr{E}}\right) \subset Z\right\}$. But then $\left(\Gamma_{\mathscr{E}}\right)^{*}=\mathscr{C}_{\mu}-\lambda_{0}$ so indeed $g_{0}=0$. Our lemma follows from this since $\mathscr{N}(g)=\hat{\mathscr{N}}(f)$.

Now let $\lambda \in \mathscr{L}_{\mu}$. Let $\mathscr{C l}(\lambda)=\lambda+\mathscr{L}(\mathscr{B})^{\perp}$. Then $\mathscr{L}_{\mu}$ is a countable union of sets of the form $\mathscr{C}(\lambda)$. The next lemma shows, among other things that each $\mathscr{C}(\lambda)$ is a coset of $\mathscr{B}^{*} /(\mathscr{B}, *)$.

LEMma 3. Let $\lambda \in \mathscr{B}^{*}$ and suppose $\lambda$ is nonzero on $\mathscr{2}(\mathscr{B})$. Then $x \cdot \lambda=0$ iff $x \in \mathscr{L}(\mathscr{B})$. Also $\mathscr{L}(\mathscr{B})^{\perp}=\{\lambda \cdot x \mid x \in \mathscr{B}\}$ and $x \cdot \lambda=$ $\lambda \cdot x$ iff $x \in \mathscr{F}(\mathscr{B})$.

Proof. Suppose $x \cdot \lambda=0$ and $x \notin \mathscr{L}(\mathscr{B})$. Then there is an $n$ such that $x \cdot \mathscr{B}^{n}=\{0\}$ and $x \mathscr{B}^{n-1} \neq 0$. Then $x \cdot \mathscr{B}^{n-1} \subset \mathscr{Z}(\mathscr{B})$ so $\lambda\left(x \mathscr{B}^{n-1}\right) \neq 0$. This is a contradiction to $\operatorname{dim} \mathscr{Z}(\mathscr{B})=1$. The second statement is equivalent to saying $\mathscr{L}(\mathscr{B})=\bigcap_{x} \operatorname{ker} \lambda \cdot x$. This follows trivially from the first statement.

To prove the third statement, if $x$ is not in $\mathscr{X}(\mathscr{B})$ there is an $n$ such that $\left[x, \mathscr{B}^{n}\right]=0$ and $\left[x, \mathscr{B}^{n-1}\right] \neq 0$. Then $\left[x, \mathscr{B}^{n-1}\right] \subset \mathscr{2}(\mathscr{B})$ so there is a $y \in \mathscr{B}^{n-1}$ such that $\lambda([x, y]) \neq 0$. But then $(x \cdot \lambda-$ $\lambda \cdot x)(y) \neq 0$, which is a contradiction.

Next we show the "finiteness" claim in Theorem B. Let $\mathscr{V}_{\mu}=$ $\left\{\beta \in \mathscr{B}^{*}|\beta| \mathscr{F}(\mathscr{B})=\lambda \mid \mathscr{Z}(\mathscr{B})\right.$ for all $\left.\lambda \in \mathscr{C l}_{\mu}\right\}$.

Lemma $4 . \quad \mathscr{V}_{\mu}=\mathscr{B} * \lambda * \mathscr{B}$.

Proof. Consider the mapping $x \rightarrow x \cdot \lambda-\lambda \cdot x$ of $\mathscr{B}$ into $\mathscr{P}(\mathscr{B})^{\perp}$. By the lemma this mapping is surjective. Let $y=\bar{x}$. Then $x * \lambda * y=$ $\lambda+x \lambda+\lambda x+x \lambda y$. By left commutativity $x \lambda y=\lambda x y=-\lambda(x+y)$ since $x+y+x y=0$. Hence $x * \lambda * y=\lambda+x \cdot \lambda-\lambda \cdot x$.

Corollary 5. Mls is a finite union of sets of the form $\Gamma * \mathscr{C l}(\lambda), \lambda \in \mathbb{l}_{\mu}$.

Proof. Let $\lambda \in \mathscr{M}_{\mu}$. Let $\widetilde{\Gamma}=\left\{x \in \mathscr{B} \mid x * \lambda \in \mathscr{C l}_{\mu}\right\}$. Then $x \in \widetilde{\Gamma}$ iff $x *(\lambda * \mathscr{B})=x * \mathscr{L}(\lambda)$ intersects $\mathscr{C} \mu$. Hence $\widetilde{\Gamma} * \mathscr{L}(\lambda)=\mathscr{L} \mu$ by Lemma 4. $\tilde{\Gamma}$ not be a subgroup of $(\mathscr{L}, *)$. However, on $\mathscr{L}(\mathscr{B})$, 
$x * \lambda(y)=\lambda(x * y * \bar{x})$. Hence $\widetilde{\Gamma}$ is a union of right $\Gamma$ cosets. The component of 0 in $\widetilde{\Gamma}$ is $\widetilde{\Gamma}_{0}=\{x|x \cdot \lambda| \mathscr{L}(\mathscr{B})=0\}$. This is a two sided ideal of $\mathscr{B}$ and hence a normal subgroup of $(\mathscr{B}, *), \quad \Gamma * \widetilde{\Gamma}_{0}$ is a closed subgroup of $(\mathscr{B}, *)$ and the quotient $M_{0}=(\mathscr{B}, *) / \Gamma * \widetilde{\Gamma}_{0}$ is compact. The image of $\widetilde{\Gamma}$ in $M_{0}$ is a discrete, closed subset. Hence $\widetilde{\Gamma} / \Gamma * \widetilde{\Gamma}_{0}$ is compact. The image of $\widetilde{\Gamma}$ in $M_{0}$ is a discrete, closed subset. Hence $\widetilde{\Gamma} / \widetilde{\Gamma} * \widetilde{\Gamma}_{0}$ is finite. Let $x_{i}$ be a collection of coset representatives for $\tilde{\Gamma}$. Then

$$
\mathscr{H}_{\mu}=U\left(x_{i} *\left(\Gamma * \Gamma_{0}\right)\right) * \mathscr{C}(\lambda) .
$$

The spaces $\Gamma \backslash \Gamma * \mathscr{M}(\lambda)$ are conveniently describable as homogeneous spaces. Let $\Gamma_{0}=\{\gamma \in \Gamma \mid \gamma * \lambda \in \mathscr{A}(\lambda)\}=\{\gamma \in \Gamma|\gamma \cdot \lambda| \mathscr{L}(\mathscr{B})=0\}=$ $\{\gamma \in \Gamma \mid \gamma * \mathscr{C}(\gamma)=\mathscr{C}(\lambda)\}$. Then $\Gamma \backslash \Gamma * \mathscr{C}(\lambda)=\Gamma_{0} \mid \mathscr{M}(\lambda)$. From Lemma 3 , for each $\gamma \in \Gamma_{0}$ there is a $\delta \in \mathscr{B}$ such that $\gamma * \lambda=\lambda * \delta$. Let $\Delta_{\lambda}=$ $\{\delta \in \mathscr{B} \mid \gamma * \lambda=\lambda * \delta$ for some $\gamma \in \Gamma\}$. Note that $\Delta_{\lambda} \supset\{x \in \mathscr{B} \mid \lambda * x=\lambda\}$. There is a one-to-one correspondence between elements of $\Gamma_{0} \mid \mathscr{M}(\lambda)$ and $(\mathscr{B}, *) / \Delta_{\lambda}$. In fact for $\beta \in \mathscr{C}(\lambda)$ there is an $x$ such that $\beta=$ $\lambda * x$. $\quad \Gamma_{0} * \beta=\lambda *\left(x * \Delta_{\lambda}\right)$. The mapping $\Gamma_{0} * \beta \rightarrow x * \Delta_{\lambda}$ defines the correspondence. Hence we have proven the following

Proposition 6. $\Gamma \backslash \mathscr{M}_{\mu}$ is a finite union of sets of the form $\Gamma \backslash \Gamma * \mathscr{M}(\lambda)$. Each of these sets is a $(\mathscr{B}, *)$ homogeneous space.

Next we prove the fact that $\mathscr{F} \psi$ is $C^{\infty}$ on $\mathscr{A}_{\mu}$. This the hardest part of the proof of Theorems A and B. The main technique is a pair of theorems on $C^{\infty}$ vectors for representations of Lie groups due to Poulsen. Specifically, let $U$ be a continuous unitary representation of a Lie group $G$ in a Hilbert space $\mathscr{H}(U)$.

Let $C^{\infty}(U)$ be the space of differentiable vectors for $U$-i.e., $C^{\infty}(U)$ is the set of vectors $v$ for which $g \rightarrow U(g) v$ is a $C^{\infty}$ mapping of $G$ into the representation space $\mathscr{H}(U)$ of $U$.

We give $C^{\infty}(U)$ its usual topology (see [9]). Let $C^{-\infty}(U)$ be the conjugate dual space to $C^{\infty}(U)$. The scalar product on $\mathscr{H}(U)$ allows us to imbed $\mathscr{H}(U)$ in $C^{-\infty}(U) . C^{\infty}(U)$ is $U$ invariant. We set $U^{\infty}=U \mid C^{\infty}(U)$. We let $U^{-\infty}$ denote the contragredient representation. The theorem which allows us to conclude that $\mathscr{F} \psi$ is a $C^{\infty}$ function is the following beautiful result due to Poulsen ([9], Corollary 2.1).

THEOREM 7. Let $U_{1}$ and $U_{2}$ be unitary representations of the Lie group $G$. Suppose $T: C^{\infty}\left(U_{1}\right) \rightarrow C^{-\infty}\left(U_{2}\right)$ is a continuous operator which intertwines $U_{1}^{\infty}$ and $U_{2}^{-\infty}$. Then the image of $T$ is contained in $C^{\infty}\left(U_{2}\right)$ and $T$ is continuous from $C^{\infty}\left(U_{1}\right)$ to $C^{\infty}\left(U_{2}\right)$. 
Proof. Let $\beta: C^{\infty}\left(U_{1}\right) \times C^{\infty}\left(U_{2}\right) \rightarrow C$ be defined by $\beta(x, y)=$ $\langle y, T x\rangle$. Then apply Corollary 2.1 of [9] and note the comments following 2.1 in [9].

The following result, which also is a corollary of results of [9], enables us to explicitly compute $\mathscr{S} \psi$.

THEOREM 8. If either $U_{1}$ or $U_{2}$ is a finite direct sum of irreducible representations then $T$ has a unique bounded extension to an intertwining operator from $\mathscr{H}\left(U_{1}\right)$ to $\mathscr{H}\left(U_{2}\right)$.

Proof. It is known that $C^{\infty}\left(U_{i}\right)$ is reflexive. Hence $T^{*}: C^{\infty}\left(U_{2}\right) \rightarrow$ $C^{-\infty}\left(U_{1}\right) . \quad T$ has a bounded extension iff $T^{*}$ does. Hence it suffices to assume that $U_{1}$ is a finite sum of irreducible representations. Therefore, it suffices to assume that $U_{1}$ is irreducible. By Theorem 7, $T^{*}: C^{\infty}\left(U_{2}\right) \rightarrow C^{\infty}\left(U_{1}\right) . \quad T^{*} T$ is then a self intertwining operator for $U_{1}^{\infty}$. By Corollary 3.5 of [9], $T^{*} T=\lambda I$. Hence $\|T\|=|\lambda|^{1 / 2}$.

Next we describe the representations we intend to use as $U_{1}$ and $U_{2}$. First some generalities on induced representations. Let $K$ be a closed subgroup of a nilpotent Lie group $N$ and let $V$ be a unitary representation of $K$ in a Hilbert space $\mathscr{H}(V)$. Let $\mathscr{H}\left(U^{V}\right)$ denote the set of $\mathscr{H}(V)$ valued measurable maps $f$ on $N$ which satisfy

(1) $f(k x)=V(k) f(x)$ for $k \in K$ and $x \in N$.

(2) $\|f\| \in L^{2}(K \backslash N)$ relative to the canonical measure.

Let $U^{V}$ be the unitary representation of $N$ in $\mathscr{H}\left(U^{v}\right)$ defined by right translation. We shall also write $U^{V}=$ ind $(K, N, V)$. Whenever we discuss induced representations we always will be using the above realization unless otherwise stated.

Now let $U_{1}=$ ind $(\Gamma,(\mathscr{B}, *), \mathscr{H})$. It follows from theorem of [9] and the compactness of $\Gamma \backslash(\mathscr{B}, *)$ that $C^{\infty}\left(U_{1}\right)$ is precisely the space of $\mu$-primary functions of $(\mathscr{B}, *)$.

LEMMA 9. $U_{1}$ is a finite sum of irreducible representations.

Proof. Since $\Gamma \backslash(\mathscr{B}, *)$ is compact it is known that $U_{1}$ is an utmost countable sum of irreducible representations and any given irreducible representation occurs in $U_{1}$ with utmost finite multiplicity. We claim that $U_{1}$ is in fact primary. This will prove our lemma. The primarity of $U_{1}$ follows from results of Moore-Wolf and the following sublemma.

Sublemma 10. Every irreducible unitary representation $U$ of 
$(\mathscr{B}, *)$, which is nontrivial on $Z(\mathscr{B})$ is square integrable in the sense of Moore-Wolf [8].

This sublemma implies the primarity of $U_{1}$ for from Moore-Wolf [8] two square integrable representations are equivalent on $(\mathscr{B}, *)$ iff they have the same character on $Z(\mathscr{B})$. Clearly each irreducible subrepresentation of $U_{1}$ is equivalent to every other irreducible subrepresentation by this criterion. To prove the sublemma it suffices to show that for any $\lambda \in \mathscr{B}^{*}$ such that $\lambda \mid Z(\mathscr{B}) \neq 0$ we have $\lambda([x, y])=0$ for all $y$ iff $x \in Z(B)$. (See Moore-Wolf, loc. cit.) This was shown in Lemma 6 above.

To describe $U_{2}$, let $\mathscr{C}_{\mu}$ be as before. By the previous results $\Gamma \backslash \mathscr{N}_{\mu}$ is a finite union of sets of the form $\Gamma \backslash \Gamma * \mathscr{M}(\lambda)$ and each of these sets is a $(\mathscr{B}, *)$ homogeneous space. Homogeneous spaces carry invariant measures so $\Gamma \backslash \mathscr{M}_{\mu}$ has a $(\mathscr{B}, *)$ invariant measure. This measure is far from unique since $\Gamma \backslash \mathscr{A}_{\mu}$ is not necessarily connected. However, we may normalize the measure on $\Gamma \backslash \mathscr{M}_{\mu}$ as follows. $\mathscr{K}_{\mu}$ is an additive coset of the additive subgroup $\mathscr{A}_{0}$ of $\mathscr{B}^{*}$ given by $\mathscr{C}_{0}=\left\{\lambda \in \mathscr{B}^{*}|\lambda| \Gamma_{\mathscr{H}}=0\right\}$. (Recall $\Gamma_{\mathscr{L}}=\Gamma \cap \mathscr{L}(\mathscr{B})$ ). As such, $\mathscr{L}_{\mu}$ carries an essentially unique measure $d \mathscr{C}$ which is invariant under the transformation $x \rightarrow x+y-z$ for all $x, y, z \in \mathscr{C}_{\mu}$. Let $f$ be a continuous function on $\mathscr{A}_{\mu}$ with compact support. Then $\sum_{\Gamma \mathscr{L} \backslash \Gamma} f(\gamma * \lambda)=\tilde{f}(\lambda)$ defines an element of $C_{c}\left(\Gamma \backslash \mathscr{M}_{\mu}\right)$ and there is a unique choice of invariant measures $v$ on $\Gamma \backslash \mathscr{K}_{\mu}$ such that $\int_{\mathscr{N}_{\mu}} f d \mathscr{C}=$ $\Sigma_{\Gamma \backslash / \mu} \tilde{f} d v$. We assume that $v$ has been so chosen.

Let $\mathscr{H}_{2}(\mu)$ be set of measurable functions on $\mathscr{K}_{\mu}$ for which

(1) $\mu(\gamma) f(\lambda)=\exp -2 \pi i\langle\gamma, \lambda\rangle f(\gamma * \lambda) \gamma \in \Gamma$.

(2) $|f| \in L^{2}\left(\Gamma \mid \mathscr{M}_{\mu}\right)$.

Then $\mathscr{H}_{2}(\mu)$ is a Hilbert space with the obvious innerproduct. We define a representation $U_{2}$ of $(\mathscr{B}, *)$ in $\mathscr{H}_{2}(\mu)$ by setting $U_{2}(g) f(\lambda)=\exp -2 \pi i\langle\bar{g}, \lambda\rangle f(\lambda * \bar{g})$. The point of these definitions is that they mimic the invariance properties of the Fourier transform of primary functions as described in Lemma 1. In fact, if $\psi$ is a primary function, $\mathscr{T} \psi$ meets the algebraic condition necessary to define a functional on $C^{\infty}\left(U_{2}\right): \mu(\gamma) \mathscr{T} \psi(\lambda)=\exp -2 \pi i\langle\gamma, \lambda\rangle \mathscr{T} \psi(\gamma * \lambda)$, for all $\gamma \in \Gamma$. The question is, does $\mathscr{T} \psi$ have the correct rate of decay at $\infty$ to define an element of $C^{-\infty}\left(U_{2}\right)$. In fact, if we can show that $\mathscr{T} \psi \in C^{-\infty}\left(U_{2}\right)$ then $\psi \rightarrow \mathscr{T} \psi$ will define a continuous intertwining operator between $C^{\infty}\left(U_{1}\right)$ and $C^{-\infty}\left(U_{2}\right)$. By Poulsen's theorem (7 above) $\psi \rightarrow \mathscr{T} \psi$ will then be a continuous mapping of $C^{\infty}\left(U_{1}\right)$ into $C^{\infty}\left(U_{2}\right)$. It will be shown that $C^{\infty}\left(U_{2}\right)$ consists of $C^{\infty}$ functions on $\Gamma \backslash \mathscr{M}_{\mu}$ so we will have proven that $\mathscr{T} \psi$ is a $C^{\infty}$ function. Actually $C^{\infty}\left(U_{2}\right)$ is not a subset of $\mathscr{S}(\mathscr{B})$, in part because 
the elements of $C^{\infty}\left(U_{2}\right)$ are supported in $\mathscr{A}_{\mu}$. This would not cause problems if each element $f$ of $C^{\infty}\left(U_{2}\right)$ could be extended to an element $\tilde{f}$ of $\mathscr{S}\left(\mathscr{B}^{*}\right)$ for then we could define $\mathscr{T} \psi(f)=\mathscr{T} \psi(\tilde{f})$. Unfortunately, the elements of $C^{\infty}\left(U_{2}\right)$ cannot be so extended as the elements of $C^{\infty}\left(U_{2}\right)$ do not tend to zero at infinity. What we will show is the following:

Let $f \in \mathscr{S}\left(\mathscr{B}^{*}\right)$ and let $y \in \Gamma$. Set

$$
V(y) f(\lambda)=\mu(y) \exp -2 \pi i\langle y, \lambda\rangle f(y * \lambda) .
$$

Note that for $y \in \Gamma_{\mathscr{L}}=\Gamma \cap \mathscr{L}(\mathscr{B})$ and $x \in \mathscr{B}$ we have $y * x=y+$ $x, y * \lambda=\lambda$ and $\mu(y)=\exp -2 \pi i\langle y, \lambda\rangle$. It follows that as a function of $y, V(y)$ is constant on cosets of $\Gamma_{\mathscr{L}}$ in $\Gamma$. Of course $\Gamma_{\mathscr{L}} \supset Z(\mathscr{B})$ so that $\Gamma_{\mathscr{L}} \backslash \Gamma$ is discrete. Let

$$
\tau_{\mu} f=\sum_{\Gamma_{\mathscr{L} \backslash} \Gamma} V(\gamma) f \mid \mathscr{M}_{\mu}
$$

We will show that $\tau_{\mu}$ is a surjective mapping of $\mathscr{L}(\mathscr{B})$ onto $C^{\infty}\left(U_{2}\right)$. Furthermore we shall show that $\operatorname{ker} \tau_{\mu} \subset \operatorname{ker} \mathscr{T} \psi$. It will follow that we may define an element $\widetilde{\psi}$ of $C^{-\infty}\left(U_{2}\right)$ by setting $\widetilde{\psi}(\tau f)=\mathscr{T} \psi(\bar{f}) . \quad \psi \rightarrow \widetilde{\psi}$ will then be an intertwining operator from $C^{\infty}\left(U_{1}\right)$ to $C^{-\infty}\left(U_{2}\right)$.

It will then be an easy matter to show that $\mathscr{T} \psi$ is a $C^{\infty}$ function on $\mathscr{N}_{\mu}$.

To describe $C^{\infty}\left(U_{2}\right)$ let $\mathscr{M}_{\mu}=\bigcup_{i=1}^{n} \Gamma * \mathscr{M}\left(\lambda_{i}\right)$ as in Lemma 5. Let $\mathscr{K}\left(\lambda_{i}\right)$ be the set of functions in $\mathscr{K}_{2}(\mu)$ which are supported in $\Gamma * \mathscr{M}\left(\lambda_{i}\right)$. The $\mathscr{K}\left(\lambda_{i}\right)$ are mutually orthogonal, invariant subspaces of $\mathscr{K}_{2}(\mu)$ and $\mathscr{K}_{2}(\mu)=\sum_{i=1}^{n} \oplus \mathscr{K}\left(\lambda_{i}\right)$. Let $U\left(\lambda_{i}\right)$ be the restriction of $U_{2}$ to $\mathscr{K}\left(\lambda_{i}\right)$. We shall describe $C^{\infty}\left(U\left(\lambda_{i}\right)\right)$. Let $i$ be fixed and set $\lambda=\lambda_{i}$.

As commented above, $\Gamma \backslash \Gamma * \mathscr{C}(\lambda) \approx(\mathscr{B}, *) / \Delta_{\lambda}$ where $=\{\delta \in \mathscr{B} \mid \lambda * \delta=$ $\gamma^{*} \lambda$ for some $\left.\gamma \in \Gamma_{0}\right\}$. This suggests that $U(\lambda)$ should be equivalent with a representation induced from a character $\chi_{\lambda}$ of $\Delta_{\lambda}$. This is the case as we now show. First we define $\chi_{\lambda}$.

Lemma 11. For $\delta \in \Delta_{\lambda}$ let

$$
\chi_{\lambda}(\delta)=\bar{\mu}(\lambda(\delta)) \exp -2 \pi i \lambda(\delta-\gamma(\delta))
$$

where $\gamma(\delta)$ is any element of $\Gamma$ such that $\gamma(\delta) \cdot \lambda=\lambda \cdot \delta$. Then $\chi_{\lambda}(\delta)$ is a well defined character of $\Delta_{\lambda}$.

Proof. First we show that $\chi_{\lambda}$ is well defined-i.e., $\bar{\mu}(\gamma(\delta))$ $\exp -2 \pi i\langle\gamma(\delta), \lambda\rangle$ does not depend on the choice of $\gamma(\delta)$ in $\Gamma$. Let $\gamma * \lambda=\gamma^{\prime} * \lambda$ for $\gamma$ and $\gamma^{\prime}$ in $\Gamma$. Then $\left(\gamma^{\prime} * \bar{\gamma}\right) * \lambda=\lambda$ so $\gamma^{\prime} * \bar{\gamma} \in \Gamma_{\mathscr{L}}$ by Lemma 3. Let $\sigma=\gamma^{\prime} * \bar{\gamma}$. Then $\gamma^{\prime}=\sigma * \gamma=\sigma+\gamma$. By definition, 


$$
\begin{aligned}
& \mu(\sigma)=\exp -2 \pi i\langle\sigma, \lambda\rangle \text { so } \\
& \mu(\gamma) \exp -2 \pi i\langle\gamma, \lambda\rangle=\mu\left(\gamma^{\prime}\right) \exp -2 \pi i\left\langle\gamma^{\prime}, \lambda\right\rangle
\end{aligned}
$$

as claimed.

To show that $\chi_{2}$ is a character, let $\delta_{1}, \delta_{2} \in \Delta_{2}$. We may take $\gamma\left(\delta_{1} * \delta_{2}\right)=\gamma\left(\delta_{1}\right) * \gamma\left(\delta_{2}\right)$. Then $\delta_{1} * \delta_{2}-\gamma\left(\delta_{1} * \delta_{2}\right)=\delta_{1}-\gamma\left(\delta_{1}\right)+\delta_{2}-\gamma\left(\delta_{9}\right)+\delta_{1} \delta_{2}-$ $\gamma\left(\delta_{1}\right) \gamma\left(\delta_{2}\right)$. Since $\mu$ is a character on $\Gamma$, it suffices to show that $\left\langle\delta_{1} \cdot \delta_{2}, \gamma\right\rangle=\left\langle\gamma\left(\delta_{1}\right) \gamma\left(\delta_{2}\right), \lambda\right\rangle$. But $\left\langle\delta_{1} \cdot \delta_{2}, \lambda\right\rangle=\left\langle\delta_{1}, \lambda \cdot \delta_{2}\right\rangle=\left\langle\delta_{1}, \gamma\left(\delta_{2}\right) \cdot \lambda\right\rangle=$ $\left\langle\gamma\left(\delta_{2}\right) \delta_{1}, \lambda\right\rangle=\left\langle\gamma\left(\delta_{2}\right), \lambda \cdot \delta_{1}\right\rangle=\left\langle\gamma\left(\delta_{1}\right) \gamma\left(\delta_{2}\right), \lambda\right\rangle$.

THEOREM 12. $U(\lambda)$ is equivalent with the induced representation ind $\left(\Delta_{\lambda}, \chi_{\lambda},(\mathscr{B}, *)\right)$.

Proof. For $f \in \mathscr{K}(\lambda)$ let

$$
T f(x)=\exp 2 \pi i\langle\bar{x}, \lambda\rangle f(\lambda * \bar{x}) .
$$

$T f$ is a map of $\mathscr{B}$ into $C$. We claim $T f$ belongs to $\mathscr{K}$ (ind $\Delta_{\lambda}, \chi_{\lambda}$, $(\mathscr{B}, *))$. Let $\delta \in \Delta_{\lambda}$. Then

$$
T f(\delta * x)=\exp 2 \pi i\langle\bar{x} * \bar{\delta}, \lambda\rangle f((\lambda * \delta) * \bar{x}) .
$$

Now

$$
f((\lambda * \bar{\delta}) * \bar{x})=f(\gamma(\bar{\delta}) *(\lambda * \bar{x}))=\bar{\mu}(\gamma(\bar{\delta})) \exp -2 \pi i\langle\gamma(\delta), \lambda * \bar{x}\rangle f(\lambda * \bar{x}) .
$$

Also

$$
\begin{gathered}
\langle\bar{x} * \bar{\delta}, \lambda\rangle=\langle\bar{x}+\bar{\delta}+\bar{x} \bar{\delta}, \lambda\rangle=\langle\bar{x}+\bar{\delta}+\lambda(\bar{\delta}) \bar{x}, y\rangle=\langle\gamma(\bar{\delta}), \lambda * \bar{x}\rangle \\
+\langle\bar{\delta}-\lambda(\delta), \lambda\rangle+\langle\bar{x}, \lambda\rangle .
\end{gathered}
$$

Combining these equalities we see

$$
T f(\delta * x)=\chi_{\lambda}(\delta) T f(x) .
$$

Since the measure on $\Gamma \backslash \Gamma * \mathscr{C}(\lambda)$ is defined by the identification of $\Gamma \backslash \Gamma * \mathscr{M}(\lambda)$ with $\Delta_{\lambda} \backslash(\mathscr{B}, *), T$ is unitary. We need to show that $T$ intertwines the given representations. This, however, is a straightforward computation similar to the above.

Next we must describe the $C^{\infty}$ vectors for ind $\left(\Delta_{\lambda}, \chi_{\lambda},(\mathscr{B}, *)\right)=$ $U(\lambda, \chi)$. They are describable in terms of the Schwartz functions on $\mathscr{B}$. Actually our description will be valid for any connected, simply connected nilpotent Lie group. Let $N$ be any such group and let $\mathscr{N}$ be the Lie algebra of $N$. Let $\mathscr{T}$ be a vector subspace of the vector space $\mathscr{N}$. Let $\mathscr{S}(\mathscr{T})$ denote the Schwartz space of rapidly decreasing $C^{\infty}$ functions on $\mathscr{T}$. Let $T \subset N$ be the set $T=$ $\exp _{N} \mathscr{T}$. By $\mathscr{S}(T)$ we shall mean the set of complex functions $f$ 
on $T$ such that $f \circ \exp _{N}$ belongs to $\mathscr{S}(\mathscr{T})$. If $\mathscr{V}$ is a topological vector space, by $\mathscr{S}(T, \mathscr{V})$ we shall mean the set of functions $f: T \rightarrow \mathscr{V}$ such that $t \rightarrow\langle f(t), w\rangle$ belongs to $\mathscr{S}(T)$ for all $w \in \mathscr{V}^{*}$ $\left(\mathscr{V}^{*}\right.$ is the continuous dual of $\left.\mathscr{V}\right)$.

We shall require an intrinsic description of $\mathscr{S}(N)$. A polynomial function on $N$ is a function $p$ on $N$ such that $p \circ \exp _{N}$ is a polynomial on $N$. A right, polynomial, differential operator on $N$ is an operator $D$ expressible in the form $D=\sum p_{i} X_{i}$ where $X_{i}$ are left invariant differential operators on $N$ and $p_{i}$ are polynomial functions on $N$.

LEMMA 13. $\exp _{N}$ maps the set of polynomial differential operators on $\mathscr{N}$ surjectively onto the set of right polynomial differential operators on $N$.

Proof. Let $Y \in \mathscr{N}$. Let $D_{Y} f(x)=d /\left.d t\right|_{t=0} f(x+t Y)$. We begin by showing that there is a polynomial operator $F_{Y}$ on $N$ such that $\left(F_{Y} f\right) \circ \exp _{N}=D_{Y}\left(f \circ \exp _{N}\right)$. Let $\left\{X_{1}, \cdots, X_{n}\right\}$ be a Jordan-Hölder basis for $\mathscr{N}$-i.e., $\left[X_{i}, X_{j}\right] \in \operatorname{span}\left\{X_{1}, \cdots, X_{j-1}\right\}$ for all $i, j$. It suffices to show $F_{X_{k}}$ exists for all $j$. We proceed by induction on $j$. For $Y \in \mathscr{N}$ let $Y$ denote also the left invariant differential operator defined by $Y$. If $i=1$ we claim that $F_{X_{1}}=X_{1}$. In fact

$$
\begin{aligned}
X_{1} f \circ \exp _{N} x & =\left.\frac{d}{d t}\right|_{t=0} f\left(\exp _{N} x \exp _{N} t X_{1}\right) \\
& =\left.\frac{d}{d t}\right|_{t} f\left(\exp _{N}\left(x+t X_{1}\right)\right)=\left(D_{X_{1}} f\right) \circ \exp _{N} .
\end{aligned}
$$

Now suppose that the theorem is true for all $j_{0}<j$. There is a polynomial mapping $p$ of $\mathscr{N} \times \boldsymbol{R}$ into $\mathscr{N}$ such that $\exp _{N} \times$ $\exp _{N} t X_{j}=\exp _{N} p(x, t) . \quad$ Furthermore $p(x, t)=x+t X_{j} \bmod \operatorname{span}\left\{\chi_{1}, \cdots\right.$, $\left.\chi_{j-1}\right\}$. Hence

$$
\begin{aligned}
X_{j} f \circ \exp _{N}(x) & =\left.\frac{d}{d t}\right|_{t=0} f \circ \exp _{N} p(x, t) \\
& =D_{X_{j}}\left(f \circ \exp _{N} x\right)+\sum_{k=1}^{j-1} p_{k}(x) D_{X_{k}} f \circ \exp _{N} x \\
& =D_{X_{j}}\left(f \circ \exp _{N} x\right)+\sum_{k=1}^{j-1} p_{k}(x)\left(F_{X_{k}} f\right) \circ \exp _{N} x .
\end{aligned}
$$

We get the desired result upon solving the above for $D_{X_{j}}$.

It is now clear that $\exp _{N}$ maps polynomial differential operators on $\mathscr{N}$ to right polynomial differential operators on $N$ as the $D_{x_{j}}$ generate the algebra of polynomial operations as a module over the polynomial functions. The surjectivity of the mapping is obvious 
since it is clear that for any right polynomial operator $D$, the operator $D^{\prime}$ given by $f \rightarrow\left(D\left(f \circ \log _{N}\right) \circ \exp _{N}\right)$ is polynomial on $\mathscr{N}$.

Corollary 14. $A C^{\infty}$ function $f$ belongs to $\mathscr{L}(N)$ iff $D f$ is uniformly bounded for all right polynomial differential operators $D$.

Now let $K$ be a closed, but not necessarily connected, subgroup of $N$. Let $\chi$ be a character of $K$. Let $U^{\chi}=$ ind $(K, N, \chi)$ realized as usual (see the above comments). Let $\tau$ be the map of $\mathscr{S}(N)$ into $\mathscr{K}\left(U^{x}\right)$ given by:

$$
\tau f(x)=\int_{K} \chi\left(k^{-1}\right) f(k x) d k .
$$

Our main result on $C^{\infty}$ vectors is the following

TheOREM 15. Suppose $U^{x}$ is a finite direct sum of irreducible representations. Then $\tau$ is a surjective mapping of $\mathscr{S}(N)$ into $C^{\infty}\left(U^{\chi}\right)$

Proof. According to theorem of [9], $C^{\infty}\left(U^{x}\right)$ is the largest space $\mathscr{C}$ of $C^{\infty}$ functions which satisfies the following

(1) $f \in \mathscr{C}$ implies that $f(k x)=\chi(k) f(x)$ for all $k \in K$ and $x \in N$.

(2) $|f| \in L^{2}(K / N)$.

(3) $\mathscr{C}$ is closed under all left invariant differential operators.

From this characterization, it is clear that $\mathscr{S}(N) \subset C^{\infty}\left(U^{x}\right)$. The only problem is to prove the surjectivity. Let $L \supset K$ be the smallest closed connected subgroup of $N$ containing $K$. Let $\mathscr{L}$ be the Lie algebra of $L$ and let $\mathscr{T}$ be a vector compliment to $\mathscr{L}$ in $\mathscr{N}$. Let $T=\exp \mathscr{T}$. Then $N=L T$ and the mapping of $L \times T \rightarrow$ $N$ given by $(l, t) \rightarrow l t$ is a $C^{\infty}$ isomorphism.

Lemma 16. There is a $C^{\infty}$ function $\phi$ on $N$ such that (i) $\phi$ is independent of the $t$ variable and is compactly supported in the $l$ variable.

(ii) $\int_{K} \phi(k x) d k=1$ for all $x \in N$.

(iii) ${ }_{\phi}$ is positive.

Proof. It suffices to assume $L=N$ for we may extend a $\phi$ which is defined on $L$ to $N$ by setting $\phi(l t)=\phi(l)$. Hence $K \backslash N$ may be assumed compact. (See [1]). Let us also assume for the moment that the identity component $K_{0}$ of $K$ is trivial so $K$ is discrete. Let $\pi: N \rightarrow K \backslash N$ be the projection map. There is a finite family of open sets $U_{1}, \cdots, U_{n}$ of $N$ such that $\pi \mid U_{i}$ is a homeomorphism onto 
an open subset of $K \backslash N$ and $\pi\left(U_{i}\right)$ cover $K \backslash N$. By passing to a subordinate covering of $K \backslash N$ if necessary, we may assume that there is a partition of unity of $K \backslash N$ relative to the $\pi\left(U_{i}\right)$ - i.e., there are $C^{\infty}$ functions $h_{i}$ on $K \backslash N$ such that each $h_{i}$ is supported in $\pi\left(U_{i}\right)$ and $\sum_{i=1}^{n} h_{i}=1$. Let $\phi_{i}$ be the function which is equal to $h_{i} \circ \pi$ on $U_{i}$ and is 0 otherwise. Let $\phi=\sum_{i=1}^{n} \phi_{i}$.

We claim that $\sum_{k} \phi(k x)=1$ for all $x$. To see this note that $\sum_{K} \phi_{i}(k x)=h_{i} \circ \pi(x)$ since $k_{1} U_{1} \cap k_{2} U_{2}=\phi \Rightarrow k_{1}=k_{2}$. Hence $\sum_{k} \phi(k x)=$ $\sum_{K} h_{i} \circ \pi=1$.

If $K_{0} \neq\{e\}$, then $K_{0}$ is normal. We form the $\phi$ corresponding to $K_{0} \backslash K$ on $K_{0} \backslash N$. Let $\pi_{0}: N \rightarrow K_{0} \backslash N$ be the projection and let $\phi_{0}=$ $\phi \circ \pi$. There is a $C^{\infty}$ function $\psi$ with compact support on cosets of $K_{0}$ such that $\int_{K_{0}} \psi(k x) d k=1$ for all $x$. Then $\phi_{0} \psi$ is the desired $\phi$ on $N$.

We shall use $\phi$ to prove the surjectivity of $\tau$ as follows. Let $f \in C^{\infty}\left(U^{x}\right)$. Then $f$ is a $C^{\infty}$ function on $N$. We claim that $f=\tau \phi f$. In fact

$$
\begin{aligned}
\tau \phi f(x) & =\int_{K} \chi\left(k^{-1}\right) \phi(k x) f(k x) d k \\
& =\int_{K} \phi(k x) d k f(x) \\
& =f(x) .
\end{aligned}
$$

Hence we need only show that $\phi f \in \mathscr{S}(N)$. Let $L$ and $T$ be as in the above proof. Let $p$ be a polynomial function on $T$. We shall show that $\sup _{t \in T}|p(t)| \sup _{l \in L}|f(l t)|<\infty$ for all $f \in C^{\infty}\left(U^{\chi}\right)$. This will imply that $\phi f \in \mathscr{S}(N)$ for let $g: \mathscr{L} \times \mathscr{T} \rightarrow C$ be given by $g(l, t)=\phi f\left(\exp _{N} l \exp _{N} t\right)$. Let $f_{0}(l, t)=f\left(\exp _{N} l \exp _{N} t\right)$ and let $\phi_{0}(l, t)=$ $\phi\left(\exp _{N} l \exp _{N} t\right)$. The mapping $(l, t) \rightarrow \log _{N}\left(\exp _{N} l \exp _{N} t\right)$ of $\mathscr{L} \times \mathscr{T}$ into $N$ is a polynomial diffeomorphism with polynomial inverse. Hence $\phi f \in \mathscr{S}(N)$ iff $g \in \mathscr{S}(\mathscr{L} \times \mathscr{T})$. Let $D$ be a polynomial differential operator on $\mathscr{L} \times \mathscr{T}$. Then from the product rule of differentiation $D g=\sum D_{i} \phi_{0} F_{i} f_{0}$ where $D_{i}$ and $F_{i}$ are polynomial differential operators on $\mathscr{L} \times \mathscr{T}$. We may assume that the $D_{i}$ are constant coefficient operators for we may absorb the coefficient of $D_{i}$ into $F_{i}$. Then $D_{i} \phi_{0}$ is independent of the $\mathscr{T}$ variable and is compactly supported in the $l$ variable. Let $\phi_{i}\left(\exp _{N} l \exp _{N} t\right)=D_{i} \phi_{0}(l, t)$ and let $f_{i}\left(\exp _{N} l \exp _{N} t\right)=F_{i} f_{0}(l, t)$. There is a right, polynomial, differential operator $\widetilde{F}_{i}$ such that $f_{i}=\widetilde{F}_{i} f$. To show that $\phi f$ is in $\mathscr{S}(N)$, it suffices to show that $\phi_{i} \widetilde{F}_{i} f$ is bounded. We may write $\widetilde{F}_{i}=$ $\sum p_{j} X_{i}$ where $p_{j}$ are polynomial functions on $N$ and $X_{j}$ are left invariant differential operators. $X_{j} f \in C^{\infty}\left(U^{x}\right)$ for all $j$ so it suffices 
to show that $\phi_{i} p f$ is bounded for all polynomial functions $p$ and all $f \in C^{\infty}\left(U^{x}\right)$. To this end, note that $p$ may be written $p(l, t)=$ $\sum_{j=1}^{n} a_{j}(l) b_{j}(t)$ where $a_{j}$ and $b_{j}$ are polynomial functions.

If we replace $\phi_{i}$ by $\phi_{i} a_{j}$ and replace $p$ by $b_{j}$, we see that it suffices to show that $\tilde{\phi} p f$ is bounded whenever $\tilde{\phi}$ is a $C^{\infty}$ function on $N=L T$ which is compactly supported in $l$ and independent of $t$ and $p$ is any polynomial in $t$ and independent of $l$.

Thus we are reduced to proving the following

LEMma 17. If $f \in C^{\infty}\left(U^{\chi}\right)$, then

$$
\sup _{t \in T}|p(t)| \sup _{l \in L}|f(l t)|<\infty
$$

for all polynomial functions $p$ on $T$.

Proof. For $t \in T$, let $f_{t}$ denote the function $l \rightarrow f(l t)$ on $L$. If $f \in C^{\infty}\left(U^{x}\right)$ then $f_{t}$ is $C^{\infty}$ on $L$ and $\left|f_{t}\right|$ belongs to $L^{2}(K \backslash L)$ since $K \backslash L$ is compact. Hence $f_{t}$ belongs to the representation space of $V^{\chi}=$ ind $(K, L, \chi) . f_{t}$ in fact belongs to $C^{\infty}\left(V^{\chi}\right)$ for $C^{\infty}\left(V^{\chi}\right)$ consists of all $C^{\infty}$ functions $h$ on $L$ which satisfy $h(k l)=\chi(k) h(l)$ since such functions are automatically square integrable modulo $K$ due to compactness. $C^{\infty}\left(V^{x}\right)$ has two natural topologies-the usual topology as a space of $C^{\infty}$ vectors (see [9]) and the topology of uniform convergence of functions and their derivatives. By the closed graph theorem, these topologies agree on $C^{\infty}\left(V^{x}\right)$. In particular $p(t) f_{t}$ will be uniformly bounded on $L$ in $t$ if $p(t) f_{t}$ is bounded as a mapping of $T$ into $C^{\infty}\left(V^{\chi}\right)$. The mapping $t \rightarrow f_{t}$ has a representation theoretic interpretation. Note that $U^{x} \approx$ ind $\left(L, N, V^{x}\right)$ due to the theorem on inducing in stages. We may realize ind $\left(L, N, V^{\chi}\right)$ as a space of $\mathscr{K}\left(V^{x}\right)$ valued functions on $T$. Then $t \rightarrow f_{t} \in \mathscr{K}$ (ind $(L$, $\left.N, V^{x}\right)$ ) and the mapping $f \rightarrow f_{t}$ defines the intertwining operator. To finish the proof of theorem, we need to show:

LEMma 18. $C^{\infty}\left(\right.$ ind $\left.\left(L, N, V^{\chi}\right)\right)$ is $\mathscr{S}\left(T, C^{\infty}\left(V^{\chi}\right)\right)$.

Proof. By assumption $U^{x} \approx$ ind $\left(L, N, V^{x}\right)$ is a finite direct sum of irreducible representations. Hence $V^{x}$ is a finite sum of irreducible representations (otherwise $U^{\chi}$ would have an infinite family of mutually orthogonal invariant subspaces). Let $V^{x}=\sum \oplus V_{i}$ where the $V_{i}$ are irreducible and let $U_{i}=$ ind $\left(L, N, V_{i}\right)$. Then $U=\Sigma \oplus U_{i}$ and $C^{\infty}(U)=\sum \oplus C^{\infty}\left(U_{i}\right)$. It suffices to show that $C^{\infty}\left(U_{i}\right)=$ $\mathscr{I}\left(T, C^{\infty}\left(V_{i}\right)\right)$. Of course, $U_{i}$ is a finite sum of irreducible representations. However an irreducible representation of a connected subgroup of a nilpotent Lie group $N$ either induces an irreducible 
representation of $N$ or induces a representation which decomposes with infinite multiplicity. Hence $U_{i}$ is irreducible. Now we change realizations of $U_{i}$ and $V_{i}$. There is a connected subgroup $M$ of $L$ and a character $\lambda$ of $M$. such that $V_{i} \approx$ ind $(M, L, \lambda)$. Then $U_{i} \approx$ ind $(M, N, \lambda)$. Let $\mathscr{C}$ be the Lie algebra of $M$ in $\mathscr{N}$. Let $\mathscr{S}$ be a vector compliment of $\mathscr{H}$ in $\mathscr{L}$. Then $\mathscr{N}=\mathscr{M} \oplus \mathscr{S} \oplus \mathscr{T}$. Let $S=\exp _{N} \mathscr{S}$. Then $N=M S T$. We define the Schwartz class $\mathscr{S}(S T)$ by setting $f \in \mathscr{S}(S T)$ iff the function $g(s, t)=f\left(\exp _{N} s \exp _{N} t\right)$ belongs to $\mathscr{S}(\mathscr{I} \times \mathscr{T})$. It follows from results of Corwin, Greenleaf and the author that ind $(M, N, \lambda)$ can be realized in $L^{2}(S T)$ and $C^{\infty}$ (ind $\left.M, N, \lambda\right)=\mathscr{S}(S T)$, [4]. For each $f \in \mathscr{S}(S T)$ and $t \in T$ let $f_{t}(s)=f(s t)$. Then for each $t, f_{t} \in \mathscr{S}(S)$ and $t \rightarrow f_{t}$ defines a bounded element of $\mathscr{S}(T, \mathscr{S}(S))$. Now $V_{i} \approx$ ind $(M, L, \lambda)$ can be realized in $L^{2}(S)$ and $C^{\infty}\left(V_{i}\right) \approx \mathscr{S}(S)$ by the results of [4] mentioned above. The representations ind $(L, N$, ind $(M, L, \lambda))$ and ind $(M, N, \lambda)$ are equivalent and the equivalence is given by mapping $f \rightarrow\left(t \rightarrow f_{t}\right)$ as defined above. Since $t \rightarrow f_{t}$ belongs to $\mathscr{S}(T, \mathscr{S}(S))$, it follows that $C^{\infty}\left(U_{i}\right) \approx C^{\infty}$ (ind $(L, N$, ind $(M, L, \lambda)) \subset \mathscr{S}(T, \mathscr{S}(S))$. But $\mathscr{S}(S)=$ $C^{\infty}($ ind $(M, L, \lambda)) \approx C^{\infty}\left(V^{i}\right)$. Hence $C^{\infty}\left(U_{i}\right)=\mathscr{S}\left(T, C^{\infty}\left(V^{i}\right)\right)$.

CoROLlaRy 19. Let $K$ be a closed subgroup of $N$ and let $\chi$ be a character of $K$ such that ind $(K, N, \chi)=U^{\chi}$ is a finite sum of irreducible representations. Then $C^{-\infty}\left(U^{x}\right)$ may be canonically identified with the space of distributions is on $\mathscr{S}(N)$ which satisfy $\psi(k x)=\chi(k) \psi(x)$ for all $k \in K . \quad(\psi(k x)$ denotes left translation of distributions).

Proof. Let $\phi$ be a $C^{\infty}$ function of $N$ such that $\int_{K} \phi(k x) d k=1$ as in Lemma 16. Then $f \rightarrow \phi f$ defines a continuous mapping of $C^{\infty}\left(U^{\chi}\right)$ into $\mathscr{S}(N)$. Let $\psi$ be a distribution on $\mathscr{S}(N)$ which satisfies the hypothesis of the theorem. Let $\tilde{\phi}(f)=\chi(\phi \bar{f})$. (-denotes complex conjugate. Recall $C^{\infty}$ is the conjugate dual). Then $\tilde{\phi} \in$ $C^{-\infty}\left(U^{x}\right)$. We claim that $\widetilde{\psi}$ does not depend on the choice of $\phi$. Let $\phi_{0}$ be another such $\phi$ and let $f_{0}=\phi_{0} f-\phi f$. Then $\tau f_{0}=0$. We need to show that $\psi\left(\bar{f}_{0}\right)=0$. To see this let $g \in \mathscr{S}(N)$. Let $L(x) g(y)=g\left(x^{-1} y\right)$. Let $\bar{f}_{0} * g=\int_{N} \bar{f}_{0}(x) g d x$, interpreted as an $\mathscr{S}(N)$ valued integral. We shall show that this integral converges in $\mathscr{S}(N)$. Granting this for the moment we see

$$
\begin{aligned}
\psi\left(\bar{f}_{0} * g\right) & =\int_{N} \bar{f}_{0}(x) \psi(L(x) g) d x \\
& =\int_{K \backslash N} \int_{K} \bar{f}_{0}(k x) \psi(L(k x) g) d k d x=\int_{K \backslash N} \tau f_{0}(x)^{-} \psi(L(x) g) d x=0 .
\end{aligned}
$$


Here we have used $\psi(L(k x) g)=\chi(k) \psi(L(x) g)$. Hence $\psi\left(\bar{f}_{0} * g\right)=0$ for all $g$. This implies $\psi\left(\bar{f}_{0}\right)=0$ as claimed. Convergence of the $\mathscr{S}(N)$ valued integral means precisely that for all $\psi \in \mathscr{S}^{\prime}(N), \bar{f}_{0}(x) \psi(L(x) g) €$ $L^{1}(N)$. (Recall that $\mathscr{S}(N)$ is reflexive and the integral is defined by reflexivity). This will follow from the following lemma.

LEMMA 20. There is a polynomial function $p$ on $N$ depending on $\psi$ and $g$ such that $|\psi(L(x) g)| \leqq p(x)$ for all $x \in N$.

Proof. As shown above $\mathscr{S}(N)$ can be characterized as the space of functions $g$ such that $D g$ is bounded for all right polynomial differential operators $D$. For each such operator $D$ let $\rho_{D}(g)=\|D g\|_{\infty}$. The family $\left\{\rho_{D}\right\}$ defines the topology on $\mathscr{S}(N)$ so there are operators $D_{i}$ such that

$$
|\psi(g)| \leqq \sum\left\|D_{i} g\right\|_{\infty}
$$

for all $g \in \mathscr{J}(N)$. We may take $D_{i}=p_{i} X_{i}$ where $X_{i}$ is left invariant differential operator on $N$ and $p_{i}$ is a polynomial function on $N$. Then $|\psi(L(x) g)|$ is bounded by a sum of terms of the form $\left\|p_{i} L(x) X_{i} g\right\|_{\infty}=\left\|\left(L\left(x^{-1}\right) p_{i}\right) X_{i} g\right\|_{\infty}$. There are polynomial functions $q$ and $r$ on $N$ such that $p_{i}(x y) \leqq|q(x)||r(y)|$ for all $x, y$ in $N$ and all $i$. Hence $\left\|L\left(x^{-1}\right) p_{i} X_{i} g\right\|_{\infty} \leqq|q(x)|\left\|r X_{i} g\right\|_{\infty}$. Thus, $|\psi(L(x) g)| \leqq$ $\sum\left(\left\|r X_{i} g\right\|_{\infty}\right)|q(x)|$.

Now we are ready to prove our first main theorem. We refer the reader to the material following Sublemma 10 for notation.

THEOREM 21. Let $\psi$ be a $\mu$-primary function on $N$. Then $\mathscr{T} \psi$ is a $C^{\infty}$ function on $\mathscr{C}_{\mu}$. In fact there is a function $f \in \mathscr{S}\left(\mathscr{B}^{*}\right)$ such that $\mathscr{T} \psi(\lambda)=\tau_{\mu} f(\lambda)$ for all $\lambda \in \mathscr{M}_{\mu}$. The image of $\mathscr{T}$ is precisely the image of $\tau_{\mu}$.

Proof. Let $\psi$ and $\mathscr{K}_{2}(\mu)$ and $U_{2}$ be as before.

Lemma 22. $\tau_{\mu}$ is a surjective mapping of $\mathscr{S}\left(\mathscr{B}^{*}\right)$ onto $C^{\infty}\left(U_{2}\right)$.

Proof. From Lemma 5, $\mathscr{C}_{\mu}$ is a finite union of sets of the form $\Gamma * \mathscr{L}(\lambda)$ for $\lambda \in \mathscr{M}_{\mu} . \quad C^{\infty}\left(U_{2}\right)$ is the direct sum of $C^{\infty}(U(\lambda))$ where $U(\lambda)$ is (as before) the restriction of $U_{2}$ to the space of functions in $\mathscr{K}_{2}(\mu)$ supported in $\Gamma * \mathscr{L}(\lambda) . \Gamma \backslash \Gamma * \mathscr{M}(\lambda)$ is in turn isomorphic with $\Gamma_{0} \mid \mathscr{L}(\lambda)$ where $\Gamma_{0}=\{\gamma \in \Gamma|\gamma \cdot \lambda| \mathscr{L}(\mathscr{B})=0\}$. Let $f \in \mathscr{F}(\mathscr{M}(\lambda))$ and let

$$
\tau_{0} f(\lambda)=\sum_{\Gamma \mathscr{L} \backslash \Gamma_{0}} \bar{\mu}(\gamma) \exp -2 \pi i\langle\gamma, \lambda\rangle f(\gamma * \lambda)
$$


It suffices, we claim, to show that $\tau_{0}$ is a surjective mapping of $\mathscr{S}(\mathscr{C}(\lambda))$ onto $C^{\infty}(U(\lambda))$, considered as a space of functions on $\mathscr{M}(\lambda)$. In fact let $g \in C^{\infty}(U(\lambda))$, considered as a function on $\Gamma * \mathscr{C}(\lambda)$. Then, assuming that $\tau_{0}$ is surjective, there is a function $f$ in $\mathscr{S}(\mathscr{L}(\lambda))$ such that $\tau_{0} f=g \mid \mathscr{M}(\lambda)$. There is a neighborhood $U$ of $\mathscr{C}(\lambda)$ such that $U \cap \mathscr{L}\left(\lambda^{\prime}\right)=\phi$ if $\lambda^{\prime} \in \mathscr{M}(\lambda)$. $f$ may be extended to an element $f^{\prime} \in \mathscr{S}\left(\mathscr{B}^{*}\right)$ which is supported in $U$. It is easily verified that $\tau_{\mu} f^{\prime}$ is zero off of $\Gamma * \mathscr{C}(\lambda)$ in $\mathscr{M}_{\mu}$ and $\tau_{\mu} f^{\prime} \mid \Gamma * \mathscr{C}(\lambda)=g$, proving our claim.

To show the surjectivity of $\tau_{0}$ we trace through the identification of $U(\lambda)$ with ind $\left(\Delta_{\lambda}, \chi_{\lambda}, \mathscr{B}\right)=U^{\chi}$ developed above. It will turn out that $\tau_{0}$ is essentially the $\tau$ associated with $U^{x}$. In fact, for $f \in \mathscr{S}(\mathscr{C}(\lambda))$ let $T f: \mathscr{B} \rightarrow C$ be given by $T f(x)=\exp 2 \pi i\langle\bar{x}, \lambda\rangle f(\lambda * \bar{x})$. Let $\{x \in \mathscr{P} \mid \lambda * x=\lambda\}=\left(\Delta_{\lambda}\right)_{0} . \quad\left(\Delta_{\lambda}\right)_{0}$ is in fact the identity component of $\Delta_{\lambda}$. For $x \in\left(\Delta_{\lambda}\right)_{0}$ and $y \in \mathscr{B}, \lambda(y * x)=\lambda(y)+\lambda(x)$. It follows that

$$
T f(x * y)=\exp 2 \pi_{i}\langle\bar{x}, \lambda\rangle T f(y)=\chi_{\lambda}(x) T f(y)
$$

for $x \in\left(\Delta_{\lambda}\right)_{0}$. Since $\left(\Delta_{\lambda}\right)_{0}$ is connected, there is a Schwartz function $g$ such that

$$
T f(x)=\int_{\left(\Delta_{\lambda}\right)_{0}} \chi_{\lambda}\left(k^{-1}\right) g(k x) d k .
$$

We claim that $\tau g=T \tau_{0} f$. In fact

$$
\begin{aligned}
\tau g & =\sum_{\left(\dot{A}_{\bar{\lambda}\rangle} \mid{ }_{\lambda}\right.} T f(\delta x) \chi_{\lambda}(\bar{\delta}) \\
& \left.=\sum \exp 2 \pi i\langle\bar{x} * \bar{\delta}, \lambda\rangle \chi_{\lambda}(\bar{\delta}) f((\lambda * \bar{\delta}) * \bar{x})\right) .
\end{aligned}
$$

Let $\lambda * \bar{\delta}=\gamma(\bar{\delta}) * \lambda$ where $\gamma(\bar{\delta}) \in \Gamma_{0} . \quad \gamma(\bar{\delta})$ is uniquely determined modulo $\Gamma_{.}$and the mapping $\delta \rightarrow \gamma(\bar{\delta})$ gives rise to a one-to-one correspondence between $\Gamma_{i} \backslash \Gamma_{0}$ and $\left(\Delta_{\lambda}\right)_{0} \backslash_{\lambda}$. Furthermore, in the proof of Theorem 12, it was shown that

$$
\exp 2 \pi_{i}\langle\bar{x} * \bar{\delta}, \lambda\rangle=\exp 2 \pi i(\langle\gamma(\bar{\delta}), \lambda * \bar{x}\rangle+\langle\bar{x}, \lambda\rangle) \chi_{\lambda}(\delta) .
$$

Hence

$$
\tau g(x)=\exp 2 \pi i\langle\bar{x}, \lambda\rangle \sum_{\Gamma \mathscr{A} \backslash \Gamma_{0}} \exp 2 \pi i\langle\gamma, \lambda * \bar{x}\rangle f(\gamma * \lambda * \bar{x})=T \tau_{0} f(x) .
$$

It follows that the image of $\tau_{0}$ is contained in $C^{\infty}(U(\lambda))$. Conversely, let $g \mathscr{S}(\mathscr{B})$. Let

$$
g_{0}(x)=\int_{\left(\Delta_{\lambda)_{0}}\right.} \chi_{\lambda}\left(k^{-1}\right) g(k x) d x
$$

and let $f(\lambda * \bar{x})=\exp -2 \pi i\langle\bar{x}, \lambda\rangle g_{0}(\bar{x})$. On $\left(A_{\lambda}\right)_{0}, \chi_{\lambda}(x)=\exp 2 \pi i\langle x, \lambda\rangle$ so $f$ is well defined. It is easily seen that $f \in \mathscr{S}(\mathscr{C}(\lambda))$ and as 
above $T_{\tau} f=\tau g$. Hence $\tau_{0}$ is onto $C^{\infty}(U(\chi))$ as claimed.

Now let $\eta$ be a Schwartz distribution on $\mathscr{B}^{*}$ which satisfies $\eta(\gamma * \lambda)=\bar{\mu}(\gamma) \exp -2 \pi i\langle\gamma, \lambda\rangle \eta(\lambda)$ for all $\gamma \in \Gamma$.

LEMma 23. There is an element $\eta^{\prime} \in C^{-\infty}\left(U_{2}\right)$ such that $\eta(\bar{f})=$ $\eta^{\prime}\left(\tau_{\mu} f\right)$ for all $f \in \mathscr{S}\left(\mathscr{B}^{*}\right)$.

Proof. For all $\lambda \in \mathscr{M}_{\mu}$ let $\eta_{\lambda}$ be the restriction of $\eta$ to $\mathscr{C}(\lambda)$. By this we mean the following. For each $f \in \mathscr{S}(\mathscr{M}(\lambda))$ there is an element $\tilde{f} \in \mathscr{S}\left(\mathscr{B}^{*}\right)$ such that $\tilde{f}$ is zero on $\mathscr{M}_{\mu} \sim \mathscr{L}(\lambda)$. Let $\eta_{\lambda}(f)=\eta(\tilde{f})$. This is independent of the extension $\tilde{f}$ by Lemma 2 and its proof. We lift $\eta_{\lambda}$ to a distribution $\eta_{\mathscr{B}}$ on $\mathscr{F}(\mathscr{B})$ by setting

$$
\eta_{\mathscr{B}}(g)=\eta_{\lambda}\left(T^{-1} g_{0}\right)
$$

where $T$ is as above and

$$
g_{0}(x)=\int_{\left(\Delta_{x}\right)_{0}} \chi_{\lambda}\left(k^{-1}\right) g(k x) d k .
$$

Then $\eta_{\mathscr{A}}(\delta x)=\chi_{\lambda}(\delta) \eta_{\mathscr{A}}(x)$ for $x \in \mathscr{B}$. By Corollary 19, there is a functional $\eta_{\mathscr{A}}^{\prime}$ in $C^{-\infty}\left(U^{x}\right)$ such that $\eta_{\mathscr{O}}(\bar{g})=\eta_{\mathscr{B}}^{\prime}(\tau g)$. Let $\eta_{x}^{\prime}(f)=$ $\eta_{\mathscr{B}}^{\prime}(T f)$ for $f \in C^{\infty}(U(\lambda))$. Then, for $f \in \mathscr{S}\left(\mathscr{B}^{*}\right)$,

$$
\begin{aligned}
\eta_{\lambda}^{\prime}\left(\tau_{0} f\right) & =\eta_{\mathscr{B}}^{\prime}\left(T \tau_{0} f\right) \\
& =\eta_{\mathscr{B}}^{\prime}(\tau g) \\
& =\eta_{\mathscr{F}}(g) \\
& =\eta_{\lambda}(f)
\end{aligned}
$$

where $g$ is any element of $\mathscr{S}(\mathscr{B})$ such that

$$
T f(x)=\int_{\left(\Delta_{x}\right)_{0}} \chi_{\lambda}\left(k^{-1}\right) g(k x) d k .
$$

Now write $\mathscr{C}_{\mu}=\bigcap_{i=1}^{n} \Gamma * \mathscr{C}\left(\lambda_{i}\right)$. We use the isomorphism of $U_{2}$ with $\Sigma \oplus U\left(\lambda_{i}\right)$ to define an element $\eta^{\prime}$ of $C^{-\infty}\left(U_{2}\right)$ by setting $\eta^{\prime}=\sum \eta_{\lambda_{i}}$. It is easily seen that $\eta^{\prime}\left(\tau_{\mu} f\right)=\eta(\bar{f})$, as desired.

It follows from Poulsen's theorem above that $(\mathscr{T} \psi)^{\prime} \in C^{\infty}\left(U_{2}\right)$ for all $\mu$-primary functions $\psi$ since $\psi \rightarrow(\mathscr{T} \psi)^{\prime}$ is a $C^{\infty}$ intertwining operator.

Lemma 24. $\mathscr{T} \psi \in C^{\infty}\left(\mathscr{M}_{\mu}\right)$ and $(\mathscr{T} \psi)^{\prime}=\mathscr{T} \psi \mid \mathscr{M}_{\mu}$.

Proof. Let $\left(\mathscr{T}^{\prime}\right)^{\prime}=g \in C^{\infty}\left(U_{2}\right)$. Let $h \in C^{\infty}\left(\mathscr{A}_{\mu}\right)$ and suppose $h$ has compact support. Since $\mathscr{H}_{\mu}$ is a countable union of affine subspaces, we may define Lebesgue measure on $\mathscr{M}_{\mu}$. Let $d x$ denote 
this measure. Let

$$
\langle g, h\rangle=\int_{\mathscr{N}_{\mu}} \bar{h} g d x .
$$

Then it can be seen that

$$
\langle g, h\rangle=\int_{\Gamma \backslash M_{\mu}}\left(\begin{array}{c}
\sum \bar{h}(\gamma * \lambda) g(\gamma * \lambda) \\
\Gamma_{\mathscr{C}} \backslash \Gamma
\end{array}\right) d v
$$

Using the invariance property of $g$ this is seen to the $\left(g, \tau_{\mu} h\right)$ where $($,$) indicates the \mathscr{C}_{2}(\mu)$ innerproduct. But by definition

$$
\left(\tau_{\mu} h, g\right)=(\mathscr{T} \psi)^{\prime}\left(\tau_{\mu} h\right)=\mathscr{T} \psi(\bar{h}) .
$$

Hence $\mathscr{T} \psi^{\prime}$ is integration against $g$, as claimed.

Next we prove surjectivity. Let $g \in C^{\infty}\left(U_{2}\right)$. Then $g \in C^{\infty}\left(\mathscr{C}_{\mu}\right)$ and $g$ is bounded on $\mathscr{L}_{\mu}$ since $g$ is in the image of $\tau_{\mu}$.

We consider $g$ as a distribution on $\mathscr{B}^{*}$ given by integrating over $\mathscr{C l}_{\mu}$ against $g d x$ where $d x$ is as above. As a distribution, $g d x$ has an inverse Fourier transform, say $\mathscr{T}^{-1}(g d x)=\psi^{\prime} \in \mathscr{S}(\mathscr{B})$. It is easily verified that $\psi$ satisfies $\psi(\gamma * x)=\mu(\gamma) \psi(x)$ for all $\gamma \in \Gamma$. Hence $\psi$ may be considered as an element of $C^{-\infty}\left(U_{1}\right)$. Hence, as above, $\mathscr{T}^{-1}$ maps $C^{\infty}\left(U_{2}\right)$ into $C^{\infty}\left(U_{1}\right) . \mathscr{T}^{-1}$ is the inverse of $\mathscr{T}$ so $\mathscr{T}$ maps $C^{\infty}\left(U_{1}\right)$ one-to-one and onto $C^{\infty}\left(U_{2}\right)$.

\section{Examples.}

EXAMPLE 1. In this section we describe a specific class of algebras from which our main examples come. Our class is motivated by Howe's work [6]. Let $\mathscr{F}$ be an abelian, nilpotent algebra which carries a nondegenerate symmetric form $B$ which satisfies $B(x y, w)=$ $B(y, x w)$. Let $\tau_{0}: \mathscr{I} \rightarrow \mathscr{J}$ be a mollifier (see $\S$ I above). Let $\mathscr{A}=$ $\mathscr{I} \times \mathscr{I}$ with the algebra structure $\left(x_{1}, x_{2}\right) \cdot\left(y_{1}, y_{2}\right)=\left(x_{1} y_{1}, x_{1} y_{2}\right)$. We shall adopt the notation that if $x \in \mathscr{A}$, then the $i$ th component of $x$ will always be denoted by $x_{i}$. On $\mathscr{A}$ let $\phi$ be the form

$$
\dot{\phi}(x, y)=B\left(x_{1}, y_{2}\right)-B\left(y_{1}, x_{2}\right) .
$$

It is easily seen that $\dot{\phi}$ satisfies the "twine" idenities:

$$
\phi(x y, w)=\dot{\phi}(y, x w)=\dot{\phi}(x,[y, w]) .
$$

$\phi$ is a nondegenerate, bi-linear form on $\mathscr{A}$ and $\mathscr{A}$ is a left communtative algebra. Let $\tau: \mathscr{A} \rightarrow \mathscr{A}$ be the form

$$
\tau x=\left(\tau_{0} x_{1},\left(I-\tau_{0}\right) x_{2}\right) .
$$


Let $\tau^{\phi}=I-\tau$.

Then $\tau$ is a left, $\mathscr{A}$-module homomorphism of $\mathscr{A}$ into $\mathscr{L}(\mathscr{A})$ which satisfies

$$
\phi(\tau x, y)=\dot{\phi}\left(x, \tau^{\phi} y\right)
$$

for all $x, y \in \mathscr{A}$. Let $\alpha(x, y)=\phi(x, \tau y)$.

LEMMA 25. Let $\mathscr{B}=\mathscr{A} \times \boldsymbol{R}$ with the product $(x, s)(y, t)=$ $(x y, \alpha(x, y))$. Then $\mathscr{B}$ is a HAT algebra. Furthermore $[(x, s),(y, t)]=$ $([x, y], \phi(x, y))$.

Proof. Associativity and left commutativity of $\mathscr{B}$ are equivalent to the identities

$$
\alpha(x y, w)=\alpha(y x, w)=\alpha(x, y w) .
$$

These follow easily from the twine identities and the properties of $\tau$. The Lie bracket identity is equivalent with $\phi(x, y)=\alpha(x, y)-$ $\alpha(y, x)$ which follows from the fact that $I-\tau$ is the adjoint of $\tau$ relative to $\phi$. It is now easily seen that $Z(\mathscr{B})=0 \times \boldsymbol{R}$.

We shall use similar direct product notation for elements of $\mathscr{B}=\mathscr{A} \times \boldsymbol{R}-$ e.g., if $x \in \mathscr{B}$, then $x=\left(x_{1}, x_{2}\right)$ where $x_{1} \in \mathscr{A}$ and $x_{2} \in \boldsymbol{R}$. Note that under our conventions $x_{1}=\left(\left(x_{1}\right)_{1},\left(x_{1}\right)_{2}\right)$ where $\left(x_{1}\right)_{i} \in$ $\mathscr{J}$. We shall seldom use this notation however.

Now, suppose $\Lambda$ is a vector lattice in $\mathscr{F}$ such that $\Lambda \cdot \Lambda \subset \Lambda$. Let us also suppose that $\tau_{0}: \Lambda \rightarrow \Lambda$. Let $\Lambda^{*}=\{x \in \mathscr{S} \mid B(\Lambda, x) \subset Z\}$. Let $\Lambda_{0}=\Lambda \times \Lambda^{*} \subset \mathscr{A}$ and let $\Gamma=\Lambda_{0} \times \boldsymbol{R} \subset \mathscr{B}$. Then $\Gamma$ is co-compact subgroup of $(\mathscr{B}, *)$. Let $\mu$ be the character of the subgroup $\Gamma$ defined by $\mu(x)=\exp 2 \pi i x_{2} . \quad \mu$ is a character because $\alpha(\Lambda, \Lambda) \subset Z$.

Lemma 26. $U^{\mu}=\operatorname{ind}\left(\Gamma,\left(\mathscr{B},{ }^{*}\right), \mu\right)$ is irreducible.

Proof. We assume that the reader is familiar both with Kirillov theory [7] and the results of [5] or [10]. Let $\lambda \in \mathscr{B}^{*}$ be defined by $\lambda(x)=x_{2}$. Let $\mathscr{C}=(0 \times \mathscr{J}) \times \boldsymbol{R} \subset \mathscr{B}$. $\mathscr{M}$ is an ideal of $\mathscr{B}$ which satisfies $\mathscr{M}^{2}=\{0\}$. It is easily seen that as a Lie algebra, $\mathscr{C}$ is an abelian, maximal subordinate subalgebra for $\lambda$ and $\lambda$ is integer valued on $\Gamma \cap \mathscr{C}$. The mapping $\exp _{\mathscr{G}}$ from $\mathscr{B}$ to $(\mathscr{B}, *)$ is $x \rightarrow x+x^{2} / 2+\cdots+x^{n} / n !+\cdots$ so on $\mathscr{M}$, $\exp _{\mathscr{B}}$ is the identity map. Hence the character corresponding to $\lambda$ on $\mathscr{M}$ is

$$
\chi(m)=\exp 2 \pi i\langle m, \lambda\rangle .
$$

From Kirillov theory ind $(\mathscr{M},(\mathscr{B}, *), \chi)=U^{\chi}$ is irreducible. Since 
$(\mathscr{B}, *)$ has square integrable representations (Lemma 10$), U^{*}$ is determined by its character on $Z(\mathscr{B})=0 \times \boldsymbol{R}$. $U^{\mu}$ has the same character as $U^{x}$ on $Z(\mathscr{B})$ so $U^{\mu}$ is a direct sum of copies of $U^{x}$. $U^{\mu}$ may in fact be identified with the $U^{x}$ primary summand of ind $(\Gamma,(\mathscr{B}, *), 1)$. The multiplicity of $U^{x}$ in $U^{\mu}$ is given by formulas in [10]. Let $x \in \mathscr{B}$ and let $\chi_{x}(m)=\chi(x * m * \bar{x})$. Since $\mathscr{M}$ is normal in $(\mathscr{B}, *), \chi_{x}$ is a character of $\mathscr{M}$. Let

$$
X=\left\{x \in \mathscr{B} \mid \chi_{x}(\Gamma \cap \mathscr{C}) \equiv 1\right\} \text {. }
$$

Then $X$ is a finite union of $\Gamma * \mathscr{C}$ cosets and the multiplicity of $U^{\chi}$ in $U^{\mu}$ is the number of such cosets. However, note that $x * y * \bar{x}=$ $x+\bar{x}+x \bar{x}+x y+y \bar{x}+x y \bar{x}=y+x y+y(\bar{x}+x \bar{x})=y+x y-y x=$ $y+[x, y]$. Hence $\chi_{x}(y)=\chi(y) \exp 2 \pi i \quad \phi\left(x_{1}, y_{1}\right)$. Thus $x \in X$ iff $Z \supset$ $\phi\left(x_{1}, \Lambda_{0} \cap(0 \times \mathscr{J})\right)=B\left(\left(x_{1}\right)_{1}, \Lambda^{*}\right)$. Hence $\left(x_{1}\right)_{1} \in \Lambda$ so $X=\Gamma^{*} \mathscr{M}$. Hence the multiplicity is one so $U^{\mu}$ is irreducible.

Now to explicitly describe the Fourier transform, we use $\phi$ to identify $\mathscr{B}$ with $\mathscr{B}^{*}$. Specifically, for $y \in \mathscr{B}$ we let $y^{*}$ denote the functional defined by

$$
\left\langle x, y^{*}\right\rangle=\phi\left(x_{1}, y_{1}\right)+x_{2} y_{2} .
$$

LEMMA 27. $x \cdot y^{*}=\left(x_{1} y_{1}-y_{2} \tau^{\phi} x_{1}, 0\right)^{*}$

$$
y^{*} \cdot x+\left(\left[x_{1}, y_{1}\right]+y_{2} \tau x_{1}, 0\right)^{*} \text {. }
$$

Proof.

$$
\begin{aligned}
\left\langle x w, y^{*}\right\rangle & =\phi\left(x_{1} w_{1}, y_{1}\right)+\alpha\left(x_{1}, w_{1}\right) y_{2} \\
& =\phi\left(w_{1}, x_{1} y_{1}\right)-\phi\left(w_{1}, y_{2} \tau^{\phi} x_{1}\right) \\
& =\left\langle w_{1},\left(x_{1} y_{1}-y_{2} \tau^{\phi} x_{1}, 0\right)^{*}\right\rangle .
\end{aligned}
$$

Hence $x y^{*}=\left(x_{1} y_{1}-y_{2} \tau^{\phi} x_{1}, 0\right)^{*}$. Similarly

$$
y^{*} \cdot x=\left(\left[x_{1}, y_{1}\right]+y_{2} \tau x_{1}, 0\right)^{*} \text {. }
$$

Now let $\lambda=(0,1)^{*}$.

Since $U^{\mu}$ is irreducible, $\Gamma \backslash \mathscr{M}_{\mu}$ is connected so $\Gamma \backslash \mathscr{C}_{\mu}=\Gamma_{0} \mid \mathscr{M}(\lambda)$ where $\Gamma_{0}=\{\gamma \in \Gamma|\gamma \cdot \lambda| \mathscr{L}(\mathscr{B})=0\}$. From Lemma 3, $\mathscr{C}(\lambda)=\lambda^{*} \mathscr{B}=$ $\left\{(\tau x, 1)^{*} \mid x \in \mathscr{A}\right\}$. Recall $\Delta_{2}=\{\delta \in \mathscr{B} \mid \lambda \cdot \delta=\gamma \cdot \lambda$ for some $\gamma \in \Gamma\}$. Hence $\Delta_{\lambda}=\tau^{-1}\left(\tau^{\phi}\left(\Lambda \times \Lambda^{*}\right)\right) \times \boldsymbol{R}$. For $\delta \in \Delta_{\lambda}$, let $\gamma_{1}(\delta)$ be any element of $\left(\tau^{\phi}\right)^{-1}\left(\tau\left(\delta_{1}\right)\right) \cap\left(\Lambda \times \Lambda^{*}\right)$ and let $\gamma(\delta)=\gamma_{1}(\delta) \times 0$. The character $\chi_{\lambda}$ of $\Delta_{2}$ is described by

$$
\begin{aligned}
\chi_{\lambda}(\delta) & =\mu(\gamma(\delta)) \exp 2 \pi i\langle(\delta-\gamma(\delta)), \lambda\rangle \\
& =\exp 2 \pi i \delta_{2} .
\end{aligned}
$$

Let $\Omega: \mathscr{H}\left(U^{\mu}\right) \rightarrow \mathscr{H}\left(U^{\alpha_{\lambda}}\right)$ be an intertwining operator. The Fourier transform $\mathscr{T}$ of a $\mu$-primary function $g$ is described by 


$$
\mathscr{T} g(\lambda * x)=C \exp -2 \pi i\langle x, \lambda\rangle \Omega f(\bar{x})
$$

from Theorem 21. Let $\tilde{\tau}: \tau(\mathscr{A}) \rightarrow \mathscr{A}$ be a linear mapping such that $\tau \widetilde{\tau}(x)=x$ for all $x \in \tau(\mathscr{A})$. Then $(x, 1)^{*}=\lambda^{*}(\tau x, 0)$ so this formula may be written as

$$
\mathscr{T} g\left((x, 1)^{*}\right)=C \Omega g\left((\widetilde{\tau} x, 0)^{-}\right) \text {. }
$$

The special case of $\tau_{0}=0$ should be mentioned. In this case $\Delta_{\lambda}$ is the connected subgroup $\mathscr{P}_{0}=(\mathscr{S} \times 0) \times \boldsymbol{R}$, and $\chi_{\lambda}(p)=\exp 2 \pi i p_{2}$. The intertwining operator from $U^{\mu}$ to ind $\chi^{\lambda}$ is known by [4]. It is, for $f \in C^{\infty}\left(U^{\mu}\right), T f(x)=\int_{\Gamma \subset \mathscr{S} \backslash \mathscr{S}_{0}} \chi_{\lambda}(p)^{-} f(p * x) d p$. This then describes the Fouries transform. Note that in this case $\tau(\mathscr{A})=0 \times \mathscr{S}$ and $\tilde{\tau}$ can be chosen to be the identity map. Furthermore $((0, x), 0)^{-}=$ $((0,-x), 0)$. Hence

$$
\mathscr{T} f\left(((0, x), 1)^{*}\right)=\int_{\mathscr{O}_{0} \cap \Gamma \mathscr{P}_{p}} f\left(p^{*} 0\right),(((0,-\bar{x}), 0)) \chi_{i} .
$$

Note also that if we identify $\mathscr{B}$ and $\mathscr{B}^{*}$ in the more "conventional" manner by setting

$$
\langle(x, t),(y, x)\rangle=B\left(x_{1}, t_{1}\right)+B\left(x_{2}, y_{2}\right)+t s .
$$

Then $((0, x), 1)^{*}=((-x, 0), 1)$ so we would write

$$
\mathscr{T}^{-} f(((x, 0), 1))=\int_{\mathscr{S}_{0} \cap T \backslash \mathscr{S}_{0}} f\left(p^{*}((0, x), 1)\right) \chi_{\lambda}(p)^{-} d p .
$$

The "flip" from $(x, 0)$ to $(0, x)$ seems very interesting.

Now let us discuss theta functions. Let $\sigma \in \boldsymbol{R}$ and let $\mathscr{P}_{\sigma}=$ $\{((s, \sigma s), t) \mid s \in \mathscr{A}, t \in \boldsymbol{R}\}$. Then $\mathscr{P}_{\sigma}$ is an abelian subalgebra of $\mathscr{B}$. Let $\chi_{\sigma}$ be the character of $\mathscr{P}_{\sigma}$ given by $\chi_{\sigma}(p)=\exp 2 \pi i \lambda\left(\log _{\mathscr{B}} p\right)$.

We shall say that a Schwartz distribution $\theta_{\sigma}$ on $\mathscr{B}$ is a $\mu$-thetadistribution if

(a) $\theta_{\sigma}(\gamma * x)=\mu(\gamma) \theta_{\sigma}(x)$ for all $\gamma \in \Gamma$

(b) $\theta_{o}(x * p)=\theta_{o}(x) \chi_{o}(p)$ for all $p \in \mathscr{P}_{\sigma}$.

The number $\sigma$ is called the period of $\theta_{\sigma}$. Note that (b) can be written

$$
\theta_{\sigma}\left(x * \exp _{\mathscr{G}} p\right)=\exp 2 \pi i \lambda(p) \theta_{o}(x) .
$$

Differentiating this equality we see that

$$
P \theta_{\sigma}=2 \pi i \lambda(P) \theta_{\sigma}
$$

for all left invariant differential operators $P$ which pass through $\mathscr{P}_{\sigma}$.

Note that from Lemma $19, \theta_{\sigma}$ gives rise to an element $\omega_{\sigma}$ of 
$C^{-\infty}\left(U^{\mu}\right)$ which satisfies $\left(U^{\mu}\right)^{-\infty}(p) \omega_{\sigma}=\chi_{\sigma}(p) \omega_{\sigma}$. Conversely, every such $\omega_{\sigma}$ gives rise to a unique $\mu$-theta distribution $\theta_{\sigma}$.

Lemma 28. For each $\sigma \in \boldsymbol{R}$ there is an essentially unique (up to scalar multiples) theta distribution $\theta_{\sigma}$.

Proof. Let $\chi_{\sigma}$ be as above. $\mathscr{P}_{\sigma}$ is a maximal subordinate subalgebra of $\mathscr{B}$ relative to $\lambda$ so the representation $U^{\sigma}=$ ind $\left(\mathscr{P}_{\sigma}\right.$, $\left.(\mathscr{B}, *), \chi_{\sigma}\right)$ is irreducible and equivalent with $U^{\mu}$. Now, from Cartier's $C^{\infty}$-Frobenius reciprocity theorem, the number of times $U^{\mu}$ occurs in $U^{\sigma}$ is the dimension of the space of elements $\omega \in C^{-\infty}\left(U^{\mu}\right)$ which satisfy $U^{\mu}(p) \omega=\chi_{\sigma}(p) \omega$. ([3], theorem). Since the multiplicity is one, we're done.

We would like to explicitly describe $\theta_{\sigma}$. To this end let $\mathscr{C}$ and $\chi$ be as in the proof of Lemma 26 above. Let $U^{x}=$ ind $(\mathscr{C}$, $(\mathscr{B}, *), \lambda)$. It is a simple matter to construct an element $\omega$ of $C^{-\infty}\left(U^{\chi}\right)$ such that $U^{\chi}(p) \omega=\chi_{\sigma}(p) \omega$. Once we have constructed such a $\omega_{\sigma}$ we shall construct an intertwining operator $T$ from $U^{\chi}$ to $U^{\mu}$. Then the image of $\omega$ under $T$ will be $\omega_{\sigma}$. To construct $\omega$, recall that $C^{-\infty}\left(U^{x}\right)$ can be cannonically identified with the space of Schwartz distributions $\psi$ which satisfy

$$
\psi(m * x)=\chi(m) \psi(x) \text { for all } m \in \mathscr{C} .
$$

Let $\omega$ be the distribution defined by the function $\omega$ defined by

$$
\omega(m * p)=\chi(m) \chi_{\sigma}(p) \quad m \in \mathscr{C}, p \in \mathscr{P}_{\sigma} .
$$

Note that $\mathscr{L} * \mathscr{P}_{\sigma}=\mathscr{B}$ and on $\mathscr{C} \cap \mathscr{P}_{\sigma}, \chi$ and $\chi_{\sigma}$ agree so this does define a function $\omega$.

Next we need to describe $T$. This is easy due to results [16] and [4]. For $f \in C^{\infty}\left(U^{x}\right)$, the function $\gamma \rightarrow f(\gamma * x)$ is constant on cosets of $\Gamma \cap \mathscr{L}$ in $\Gamma$ for each $s$. Let

$$
T f=\sum_{\Gamma \cap \mathscr{A} \backslash \Gamma} f(\gamma * x) .
$$

Then by [4], this sum converges uniformly on compact subsets to an element of $C^{\infty}\left(U^{\mu}\right) . \quad T$ is the desired intertwining operator. The image of $\omega$ under $T$ (i.e., $\left.\omega \circ T^{*}\right)$ is then the element of $C^{-\infty}\left(U^{\mu}\right)$ defined by the distribution

$$
\theta_{\sigma}(x)=\sum_{\Gamma \cap \mathscr{N} \Gamma \Gamma} \omega(\gamma * x) .
$$

This sum converges in the sense of distributions.

Next we would like to compute $\theta_{\sigma}$ explicitly in terms of the 
"coordinates" for $\mathscr{B}$. Since $\theta_{\sigma}((w, t))=\exp 2 \pi i t \theta_{\sigma}((w, 0))$, it suffices to compute $\theta_{\sigma}(w, 0)$. First we must compute $\chi_{\sigma}$ which means computing $\chi_{o} . \chi_{o}$ involves $\log _{\mathscr{O}}$ so we compute $\log _{\mathscr{O}}(w, 0)$ first. We begin by noting that $\alpha\left((x, y),\left(x, y^{\prime}\right)\right)=\phi\left((x, y), \tau\left(x^{\prime}, y^{\prime}\right)\right)=\phi((x, y)$, $\left.\left(\tau_{0} x^{\prime}\left(I-\tau_{0}\right) y^{\prime}\right)\right)=B\left(x,\left(I-\tau_{0}\right) y^{\prime}\right)-B\left(y, \tau_{0} x^{\prime}\right)$. Now by induction it is seen that for $n \geqq 2$,

$$
\begin{aligned}
((x, y), 0)^{n} & =\left(\left(x^{n}, x^{n-1} y\right), \alpha\left((x, y),\left(x^{n-1}, x^{n-2} y\right)\right)\right) \\
& =\left(\left(x^{n}, x^{n-1} y\right), B\left(x,\left(I-\tau_{0}\right) x^{n-2} y\right)-B\left(y, \tau_{0} x^{n-1}\right)\right) .
\end{aligned}
$$

If $n=2$ this says

$$
((x, y), 0)^{2}=\left(\left(x^{2}, x y\right),-2 B\left(\tau_{0} x, y\right)+B(x, y)\right) .
$$

If $n>2$, then $\tau_{0} x^{n-1}=0$ since $\tau_{0} \mathscr{J}^{2}=0$. Thus $((x, y), 0)^{n}=\left(\left(x^{n}, x^{n-1} y\right)\right.$, $\left.B\left(x, x^{n-2}\right)\right)$. Now suppose $y=\sigma x$. Let

$$
l(x)=\sum_{n=2}^{\infty}(-1)^{n+1} B\left(x, x^{n-1}\right) / n .
$$

The following lemma is easily proven from the above comments.

\section{LEMMA 29.}

$$
\log _{\mathscr{P}}((x, \sigma x), 0)=\left(\left(\log _{\mathscr{f}} x, 6 \log _{\mathscr{L}} x\right), \sigma B\left(\tau_{0} x, x\right)+\sigma l(x)\right) .
$$

Hence $\chi_{o}((x, \sigma x), 0)=\exp 2 \pi i \sigma\left(B\left(\tau_{0} x, x\right)+l(x)\right)$. Now let $((x, y), 0) \in$ $\mathscr{B}$. Then $((x, y), 0)=((0, y-\sigma x),-\alpha((0, y-\sigma x),(x, \sigma x)))^{*}((x, \sigma x), 0)=$ $\left((0, y-\sigma x), B\left(y-\sigma x, \tau_{0} x\right)\right) *((x, \sigma x))$. Hence

$$
\begin{aligned}
\omega((x, y), 0) & =\exp 2 \pi i\left(B\left(y-\sigma x, \tau_{0} x\right)+\sigma B\left(\tau_{0} x, x\right)+\sigma l(x)\right) \\
& =\exp 2 \pi i\left(B\left(y, \tau_{0} x\right)+\sigma l(x)\right) .
\end{aligned}
$$

Now the set $A_{1}=\{((n, 0), 0) \mid n \in \Lambda\}$ is a complete set of inequivalent representatives of the $\Gamma \cap \mathscr{C}$ cosets in $\Gamma$. Given $w=$ $((x, y), 0)$ in $\mathscr{B}$ and $\gamma=((n, 0), 0)$ in $A_{1}$,

$$
\gamma * w=\left((n * x, y+n y), B\left(n,\left(I-\tau_{0}\right) y\right)\right) .
$$

Now, note that $\tau_{0}(n * x)=\tau_{0}(n+x)$ since $\tau_{0} \mathscr{J}^{2}=0$. Hence,

$$
\begin{aligned}
\mathscr{B}\left(y+n y, \tau_{0}(n * x)\right) & =\mathscr{B}\left(y+n y, \tau_{0}(n+x)\right) \\
& =\mathscr{B}\left(\tau_{0}(y+n y), n+x\right)=\mathscr{B}\left(\tau_{0} y, n+x\right) .
\end{aligned}
$$

Hence

$$
\begin{aligned}
\omega(\gamma * w) & =\exp 2 \pi i\left(\left(\tau_{0} y, x+n\right)+\sigma l(n * x)+B\left(n,\left(I-\tau_{0}\right) y\right)\right) \\
& =\exp 2 \pi i\left(B\left(\tau_{0} y, x\right)+B(n, y)+\sigma l(n * x)\right) .
\end{aligned}
$$

Finally, then 


$$
\theta_{\sigma}(w)=\exp 2 \pi i B\left(\tau_{0} y, x\right) \sum_{n \in A} \exp 2 \pi i(B(n, y)+\sigma l(n * x)) .
$$

We simplify this by means of the following lemma which follows easily from Lemma 29 above with $\tau_{0}=0$.

Lemma 30. For all $x, y \in \mathscr{J}, l\left(x^{*} y\right)=l(x)+l(y)-B(x, y)$. It follows from this lemma that

$$
\theta_{\sigma}(w)=\exp 2 \pi i\left(B\left(\tau_{0} y, x\right)+l(x)\right) \sum \exp 2 \pi i(B(n, y-\sigma x)+\sigma l(n)) .
$$

This is exactly the mollified theta series of $\S I$.

To prove the theta identity we re-compute $\theta_{\sigma}$ in the $\tau_{0}=0$ case. We follow the same procedure as above except that we use the subgroup $\mathscr{P}_{0}$ and the character $\chi_{0}$ instead of the pair $(\mathscr{C}, \chi)$. Let $\omega_{0}\left(p_{0} * p_{1}\right)=\chi_{0}\left(p_{0}\right) \chi_{\sigma}\left(p_{1}\right)$ for $p_{0} \in \mathscr{P}_{0}, p_{1} \in \mathscr{P}_{\sigma}$. Then from the uniqueness of $\theta_{\sigma}$

$$
\theta_{\sigma}(w)=K(\sigma) \sum_{\mathscr{O}_{0} \cap \backslash \backslash \Gamma} \omega_{0}(\gamma * w) .
$$

To explicitly compute $\omega_{0}$, observe that $((x, y), 0)=((u, 0), 0) *((v$, $\sigma v), t)$ where $u=x-\sigma^{-1} y, v=x * \bar{u}$ and $t=-\sigma B(u, v)$. Then $\omega_{0}(w)=$ $\exp 2 \pi i \sigma(l(v)-B(u, v))$. We simplify using the above lemma.

$$
\begin{aligned}
l(v) & =l(x)+l(\bar{u})-B(x, \bar{u}) . \\
0 & =l(u * \bar{u})=l(u)+l(\bar{u})-B(u, \bar{u}) .
\end{aligned}
$$

$B(u, v)=B(u, x+x \bar{u}+\bar{u})=B(u+u \bar{u}, x)+B(u, \bar{u})=B(\bar{u}, x)+B(u, \bar{u})$. Hence

$$
\begin{aligned}
\omega_{0}(w) & =\exp 2 \pi i \sigma(l(x)-l(u)) \\
& =\exp 2 \pi i \sigma\left(l(x)-l\left(x-\sigma^{-1} y\right)\right) .
\end{aligned}
$$

Hence we see that

$$
\theta_{\sigma}(w, 0)=K(\sigma) \exp 2 \pi i \sigma l(x) \sum_{n \in A} \exp 2 \pi i \sigma l\left(\sigma^{-1}(\sigma x-y+n)\right) .
$$

Equating the above expression of $\theta_{\sigma}$ with our first expression and replacing $\sigma x-y$ by $x$ we see:

THEOREM 31.

$$
\begin{aligned}
& \sum \exp 2 \pi i(\sigma l(n)+B(x, n)) \\
& \quad=\sum \exp 2 \pi i \sigma l\left(\sigma^{-1}(x+n)\right) .
\end{aligned}
$$

This is the desired theta identity.

REMARK. It was originally our hope that one could use the 
generalized theta series to compute sums of the form $\sum_{n=0}^{p-1} \exp 2 \pi i n^{k} / p$ where $p$ is some integer. Formulas for such sums are known classically only for $0 \leqq k \leqq 2$. The relevance of the theta series is as follows. Let $\mathscr{J}_{Q}=\operatorname{span}_{Q} \Lambda$. Let $x \in \mathscr{J}_{Q}$ and let $\sigma \in Q$. It is easily verified that there is a sub-lattice $\Lambda_{0} \subset \Lambda$ such that the functions $\exp 2 \pi i B(n, x)+\sigma l(n)$ and $\exp 2 \pi i \sigma l\left(\sigma^{-1}(x+n)\right)$ are both constant on coset of $\Lambda_{0}$ in $\Lambda$ as functions of $n$. Let:

$$
\begin{aligned}
& \theta_{\sigma}^{Q}(x)=\left[\Lambda_{0}: \Lambda\right]^{-1} \sum_{\Lambda_{0} \backslash \Lambda} \exp 2 \pi i(B(n, x)+\sigma l(n)) \\
& \psi_{\sigma}^{Q}(x)=\left[\Lambda_{0}: \Lambda\right]^{-1} \sum_{\Lambda_{0} \backslash \Lambda} \exp 2 \pi i \sigma l\left(\sigma^{-1}(x+n)\right) .
\end{aligned}
$$

The function $\theta_{o}^{Q}$ represents, in some very real sense, the "values" of the distribution $\theta_{\sigma}$ at the elements of $\mathscr{F}_{Q}$. It seems reasonable to conjecture that $\theta^{Q}=K(\sigma) \psi_{\sigma}^{Q}$. Granted this, let $\sigma$ be an integer chosen so that $\sigma l(n) \in Z$ for all $n \in \Lambda$. It is easy to see that such $\sigma$ exist. Let $x=0$. Then $\theta_{\sigma}^{Q}(x)=1$ while $\psi_{\sigma}^{Q}(x)=\sum \exp 2 \pi \sigma l\left(\sigma^{-1} n\right)$. The theta identity says $\psi_{\sigma}^{Q}(0)=K(\sigma)^{-1}$. Of course $\sigma l\left(\sigma^{-1} n\right)$ can have arbitrarily large degree so we can compute some greater than quadratic sums provided we can compute $K(\sigma) . K(\sigma)$ should be computable as follows.

For $\rho$ arbitrary, let $U^{\rho}=$ ind $\left(\mathscr{P}_{\rho},(\mathscr{B}, *), \chi_{\rho}\right)$. Also set $U^{x}=$ ind $(\mathscr{M},(\mathscr{B}, *), \chi)$. Let $T^{\rho, 0}$ be the operator from $\mathscr{H}\left(U^{\rho}\right)$ to $\mathscr{H}\left(U^{0}\right)$ defined by $T^{\rho, 0} f(x)=\int_{\mathscr{P}_{\rho} \cap \mathscr{O}_{0} \mid \mathscr{S}_{0}} f(p * x) \bar{\chi}_{0}(p) d p$. It is easily verified that $T^{\rho, 0}$ defines an intertwining operator $U^{\rho}$ to $U^{0}$. Let $T^{\rho, \chi}: U^{\rho} \rightarrow U^{x}$ be defined similarly using $(\mathscr{M}, \chi)$ in place of $\left(\mathscr{P}_{0}, \chi_{0}\right) . \quad T^{\rho, 0}$ and $T^{\rho, \chi}$ may be extended to intertwining operators on the $C^{-\infty}$ spaces of the corresponding representations. Let $\delta \in C^{-\infty}(U)$ be defined by $\delta(f)=f(0)$.

Note that $\delta\left(U^{\sigma}(p) f\right)=f(p)=\chi_{\sigma}(p) f(0)=\chi_{\sigma}(p) \delta(f)$ for $p \in \mathscr{P}_{\sigma}$. It is easily verified that $T^{\sigma, 0}(\delta)=\omega_{0}$ and $T^{\sigma, \alpha}(\delta)=\omega$. Recall $\omega_{0}$ is defined by $\omega_{0}\left(p_{0} * p_{\sigma}\right)=\chi_{0}\left(p_{0}\right) \chi_{\sigma}\left(p_{\sigma}\right)$ and $\omega\left(m * p_{\sigma}\right)=\chi(m) \chi_{\sigma}\left(p_{\sigma}\right)$ for $p_{0} \in \mathscr{P}_{0}$, $p_{o} \in \mathscr{P}_{\sigma}$ and $m \in \mathscr{M}$. Let $T^{0, \chi}$ and $T^{\chi, \lambda}$ be the respective intertwining operators from $U^{0}$ and $U^{\chi}$ to $U^{\lambda}=$ ind $(\Gamma Z(\mathscr{B}, *), \lambda)$ used in the computation of $\theta_{\sigma}$ above. Then $\theta_{\sigma}=T^{x, \lambda} \omega=K(\sigma) T^{0, x} \omega_{0}$. Hence $T^{\chi, \lambda} T^{\sigma, \gamma} \delta=K(\sigma) T^{0, \lambda} T^{\sigma, 0} \delta$. From the uniqueness of intertwining operators this implies $T^{\chi, \lambda} T^{\sigma, \gamma}=K(\sigma) T^{0, \lambda} T^{\sigma, 0}$. However

$$
\begin{aligned}
T^{\chi, \lambda} T^{o, \chi} & =T^{\chi, \lambda} T^{0, \chi}\left(T^{0, \chi}\right)^{-1} T^{o, \lambda} \\
& =C T^{0, \chi}\left(T^{0, \chi}\right)^{-1} T^{o, \chi}
\end{aligned}
$$

where $C$ is a constant independent of $\sigma$.

It follows that

$$
C^{-1} K(\sigma) T^{\sigma}=\left(T^{0, x}\right)^{-1} T^{\sigma, \chi}
$$

i.e., up to constant multiple $K(\sigma)$ is the intertwining "constant" of 
$T^{\sigma, 0}, T^{0, \chi}$ and $T^{\sigma, \chi}$. Note that this constant doesn't depend on $\Gamma$ at all. It should be expressible as an integral of some sort. Presuming that the intertwining constant can be computed we can evaluate $C$ by setting $\sigma=1, x=0$ and using the theta identity which says $1=K(1) \psi_{1}^{Q}(0)=K(1) \sum \exp 2 \pi i l(n)$. This computes $K(1)$ from which $C$ can be found.

We have not been anxious to carry out the above procedure for the following reason. In every example we have tried to compute, so far, we only obtain information concerning the linear or quadratic sums, regardless of the nilpotent degree of $\mathscr{J}$. This is probably due to the tendency of nilpotent analysis to reduce to the two step case. The formulas for $K(\sigma)$ should still be computed, as the expressions for $\psi_{\sigma}(0)$ in terms of Gauss sums are complicated and a formula for them might express interesting number theoretic information. It is conceivable also that by exploiting a more general class of algebras one could obtain more information. For example, one might try making $\mathscr{I}$ non-abelian or using solvable groups instead of nilpotent groups.

Next we compute the Fourier transform of the mollified theta series. We shall do this in general only in the case that the mollifier $\tau_{0}$ is trivial. However, we shall demonstrate the effect of the mollifier by considering two special cases. One case is the Heisenberg group and the other is a case that $\mathscr{J}$ is cyclic (generated by one generator). The effect of the mollifier is quite interesting. The effect is to increase the singular support of the Fourier transform. At first glance it may seem undesirable to increase the singular support. However, recall that the Fourier transform of a $C^{\infty}$ function is a $C^{\infty}$ function times Haar measure on the singular support, and is zero off of the singular support. Hence the larger the singular support, the more the Fourier transform looks like a $C^{\infty}$ function.

Another curious fact concerning the effect of the mollifier on the Fourier transform is that the mollifier does not alter the isomorphism class of the Lie algebra. In fact a mollified algebra gives rise to precisely the same Lie algebra as the corresponding unmollified algebra. Despite this the Fourier transforms of the same function can be quite different, depending on the particular algebra chosen to represent the Lie algebra. This occurs even in the three dimensional case.

To describe the Fourier transform of the mollified theta series in the $\tau_{0}=0$ case, let $\omega_{0}$ be as before:

$$
\omega_{0}\left(p_{0} * p_{\sigma}\right)=\chi_{0}\left(p_{0}\right) \chi_{\sigma}\left(p_{\sigma}\right)
$$

From the above computations $\mathscr{T} \theta_{\sigma, 0}$ may be identified canonically 
with an element of $C^{-\infty}\left(U^{x_{2}}\right)$ where $U^{x_{2}}=$ ind $\left(\Delta_{\lambda_{1}},\left(B,{ }^{*}\right), \chi_{\lambda}\right)$. In the $\tau_{0} \equiv 0$ case, $\Delta_{\lambda}=\mathscr{P}_{0}, \chi_{\lambda}=\chi_{0}$. The uniqueness of $\theta_{\sigma, 0}$ implies the following.

THEOREM 32. There is a constant $C(\sigma)$ such that $\mathscr{T} \theta_{\sigma, 0}=C(\sigma) \omega_{0}$.

Thus, except for computing $C(\sigma)$, the Fourier transform of $\theta_{\sigma, 0}$ is automatic. We shall not attempt to compute $C(\sigma)$ as we have no need for this information at the moment.

In the mollified cases, things become more complicated.

EXAMPLE II. Let $\mathscr{I}=\boldsymbol{R}$ with trivial multiplication. Let $\Lambda=\boldsymbol{Z}$ and let $B(x, y)=x y$. Let $\tau_{0}: \mathscr{I} \rightarrow \mathscr{J}$ be the map $\tau_{0}(x)=(1 / 2) x . \tau_{0}$ is a mollifier. The mapping $\tau: \mathscr{I} \times \mathscr{I} \times \mathscr{J}$ is given by $\tau(x, y)=$ $1 / 2(x, y)$. Hence $\Delta_{\lambda}=\tau^{-1}\left(\tau^{\phi}(\Lambda \times \Lambda)\right) \times \boldsymbol{R}=\boldsymbol{Z}^{2} \times \boldsymbol{R}=\Gamma$. It is easily checked that $\chi_{\lambda}=\mu$. Hence, in this case, the Fourier transform is a scalar multiple of the identity map. Note, however that we are using an unconventional identification of $\mathscr{B}$ with $\mathscr{B}^{*}$. In more conventional terms $\mathscr{T} \psi$ is supported in $(\boldsymbol{R} \times \boldsymbol{R}) \times \boldsymbol{Z}$ and $\mathscr{T} \psi((x, y), n)=$ $C_{\psi}(\Gamma((-y, x), 0)$.

EXAMPLE III. Let $\mathscr{J}$ be generated by a single generator $e$ subject to the relation $e^{3}=0$. Let $B$ be defined by

$$
\begin{aligned}
B\left(e^{i}, e^{j}\right) & =1 & & \text { if } i+j=3 \\
& =0 & & \text { otherwise. }
\end{aligned}
$$

We extend $B$ bi-linearly to $\mathscr{F} \times \mathscr{F}$. It is easily verified that $B(x y, w)=B(x, y w)$ for all $x, y, w$. Let $\tau_{0}: \mathscr{F} \times \mathscr{F}$ be multiplication by $e . \tau_{0}$ is a mollifier. Let $\Lambda \subset \mathscr{J}$ be the span of $\left\{e^{1}, e^{2}\right\}$ over $Z$. It is easily computed (from $\tau^{-1}\left(\tau^{\phi}(\Lambda \times \Lambda)\right)$ ) that $\Delta_{\lambda}=\left\{\left(\left(x, n e^{2}\right), t\right) \mid x \in\right.$ $\mathscr{J}, m \in Z, t \in R\}$ and $\chi_{2}$ is the map $\left(\left(x, n e^{2}\right), t\right) \rightarrow \exp 2 \pi i t$. An intertwining operator from $U_{0}$ to $U^{x_{2}}$ is given by

$$
\tau f(x)=\sum_{n} f\left(\left(0, n e^{2}\right), 0^{*} x\right) .
$$

From the uniqueness of $\theta_{\sigma, \tau_{0}}$, it follows that $\mathscr{T} \theta_{\sigma, \tau_{0}}(w)=\omega_{0}\left(\left(0, n e^{2}\right)\right.$, $0)^{*}(w, 0)$. Letting $w=(x, y)$ and computing we see

$$
\begin{aligned}
\mathscr{T} \theta_{\sigma, \tau_{0}}(w) & =C(\sigma) \sum \omega_{0}\left(w+n e^{2}\right) \\
& =c(\sigma) \sum \omega_{0}\left(x, y+n e^{2}\right) .
\end{aligned}
$$

\section{REFERENCES}

1. L. Auslander, An exposition of the structure of solvmanifolds, Part I, Bull. Amer. Math. Soc., 79 (1973), 227-261. 
2. L. Auslander and R. Tolimieri, Abelian harmonic analysis, theta functions and function algebras on a nilmanifold, Lecture Notes in Mathematics, \#436, Springer, Berlin, 1975.

3. Cartier, Quantum mechanical commutation relations and Theta functions, Proceedings of the Boulder symposium on algebraic groups and discontinuous subgroups, 1966.

4. Corwin, Greenleaf, Penney, A general character formula for irreducible projections on $L^{2}$ of a nilmanifold, Math. Ann., 225 (1977), 21-32.

5. R. Howe, On Frobenius reciprocity for unipotent algebraic groups over $Q$, Amer. J. Math., 43 (1971), 163-172.

6 . On a connection between nilpotent groups and oscillatory integrals associated to singularities, Pacific. J. Math., 73 (1977), 329-364.

7. A. Kirillov, Unitary representations of nilpotent Lie groups, Russian Mathematical Surveys, 17 (1962), 53-104.

8. C. Moore and J. Wolf, Square integrable representations of nilpotent groups, Trans. Amer. Math. Soc., 185 (1973), 445-462.

9. N.S. Poulsen, On C-vectors and intertwining bilinear forms for representations of Lie groups, J. Functional Analysis, 9 (1972), 87-120.

10. L. Richardson, Decomposition of the $L^{2}$ space of a general compact nilmanifold, Amer. J. Math., 43 (1971), 173-190.

Received January 15, 1980.

Purdue UnIVERSITY

WeSt LAFAyetTe, IN 47907 


\title{
PACIFIC JOURNAL OF MATHEMATICS
}

\section{EDITORS}

\author{
DONALD BABBITT (Managing Editor) \\ University of California \\ Los Angeles, CA 90024 \\ Hugo Rossi \\ University of Utah \\ Salt Lake City, UT 84112 \\ C. C. MOORE and ARthur Agus \\ University of California \\ Berkeley, CA 94720
}

J. DUGUNDJI

Department of Mathematics

University of Southern California

Los Angeles, CA 90007

R. FINN and J. MILGRAM

Stanford University

Stanford, CA 94305

\section{ASSOCIATE EDITORS}
R. ARENS
E. F. BeCKENBACH
B. H. NeumanN
F. WoLF
K. YoSHIDA

\section{SUPPORTING INSTITUTIONS}

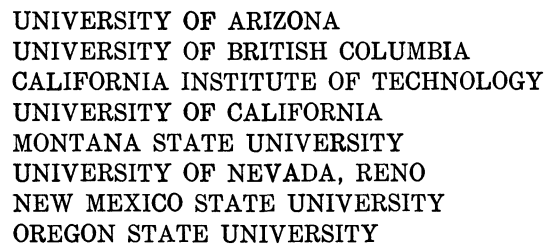

UNIVERSITY OF ARIZONA

UNIVERSITY OF BRITISH COLUMBIA

CALIFORNIA INSTITUTE OF TECHNOLOGY

UNIVERSITY OF CALIFORNIA

MONTANA STATE UNIVERSITY

UNIVERSITY OF NEVADA, RENO

NEW MEXICO STATE UNIVERSITY

OREGON STATE UNIVERSITY

\author{
UNIVERSITY OF OREGON \\ UNIVERSITY OF SOUTHERN CALIFORNIA \\ STANFORD UNIVERSITY \\ UNIVERSITY OF AAWAII \\ UNIVERSITY OF TOKYO \\ UNIVERSITY OF UTAH \\ WASHINGTON STATE UNIVERSITY \\ UNIVERSITY OF WASHINGTON
}

The Supporting Institutions listed above contribute to the cost of publication of this Journal, but they are not owners or publishers and have no responsibility for its content or policies,

Mathematical parers intended for publication in the Pacific Journal of Mathematics should be in typed form or offset-reproduced, (not dittoed), double spaced with large margins. Please do not use built up fractions in the text of the manuscript. However, you may use them in the displayed equations. Underline Greek letters in red, German in green, and script in blue. The first paragraph or two must be capable of being used separately as a synopsis of the entire paper. Please propose a heading for the odd unmbered pages of less than 35 characters. Manuscripts, in triplicate, may be sent to any one of the editors. Please classify according to the scheme of Math. Reviews, Index to Vol. 39. Supply name and address of author to whom proofs should be sent. All other communications should be addressed to the managing editor, or Elaine Barth, University of California, Los Angeles, California, 90024.

50 reprints to each author are provided free for each article, only if page charges have been substantially paid. Additional copies may be obtained at cost in multiples of 50 .

The Pacific Journal of Mathematics is issued monthly as of January 1966, Regular subscription rate: $\$ 114.00$ a year (6 Vol., 12 issues). Special rate: $\$ 57.00$ a year to individual members of supporting institution.

Subscriptions, orders for numbers issued in the last three calendar years, and changes of address shoud be sent to Pacific Journal of Mathematics, P.O. Box 969, Carmel Valley, CA 93924, U.S.A. Old back numbers obtainable from Kraus Periodicals Co., Route 100, Millwood, NY 10546.

PUBLISHED BY PACIFIC JOURNAL OF MATHEMATICS, A NON-PROFIT CORPORATION

Printed at Kokusai Bunken Insatsusha (International Academic Printing Co., Ltd.). 8-8, 3-chome, Takadanobaba, Shinjuku-ku, Tokyo 160, Japan. 


\section{Pacific Journal of Mathematics}

\section{Vol. 103, No. $2 \quad$ April, 1982}

Alberto Alesina and Leonede De Michele, A dichotomy for a class of positive

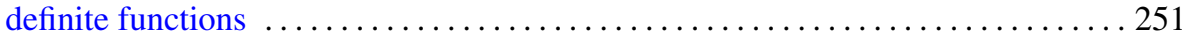

Kahtan Alzubaidy, Rank 2 -groups, $p>3$, and Chern classes . . . . . . . . . . 259

James Arney and Edward A. Bender, Random mappings with constraints on

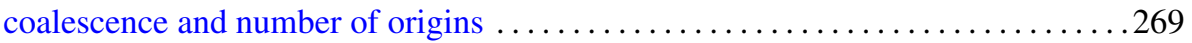

Bruce C. Berndt, An arithmetic Poisson formula . . . . . . . . . . . . . . . 295

Julius Rubin Blum and J. I. Reich, Pointwise ergodic theorems in 1.c.a. groups . . . 301

Jonathan Borwein, A note on $\varepsilon$-subgradients and maximal monotonicity . . . . . . . 307

Andrew Michael Brunner, Edward James Mayland, Jr. and Jonathan Simon,

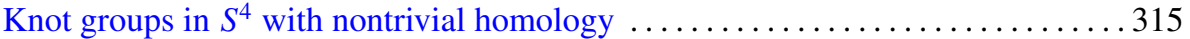

Luis A. Caffarelli, Avner Friedman and Alessandro Torelli, The two-obstacle

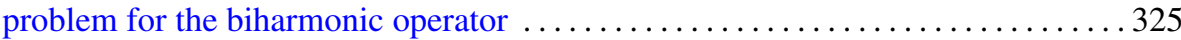

Aleksander Całka, On local isometries of finitely compact metric spaces . . . . . . 337

William S. Cohn, Carleson measures for functions orthogonal to invariant

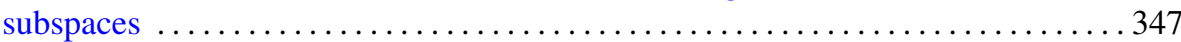

Roger Fenn and Denis Karmen Sjerve, Duality and cohomology for one-relator

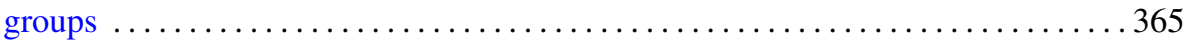

Gen Hua Shi, On the least number of fixed points for infinite complexes . . . . . . . 377

George Golightly, Shadow and inverse-shadow inner products for a class of linear

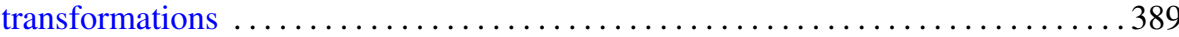

Joachim Georg Hartung, An extension of Sion's minimax theorem with an

application to a method for constrained games $\ldots \ldots \ldots \ldots \ldots \ldots \ldots \ldots \ldots \ldots . \ldots 4$

Vikram Jha and Michael Joseph Kallaher, On the Lorimer-Rahilly and

Johnson-Walker translation planes

Kenneth Richard Johnson, Unitary analogs of generalized Ramanujan sums .

Peter Dexter Johnson, Jr. and R. N. Mohapatra, Best possible results in a class of inequalities

Dieter Jungnickel and Sharad S. Sane, On extensions of nets

Johan Henricus Bernardus Kemperman and Morris Skibinsky, On the

characterization of an interesting property of the arcsin distribution ...

Karl Andrew Kosler, On hereditary rings and Noetherian $V$-rings

William A. Lampe, Congruence lattices of algebras of fixed similarity type. II . . . . 475

M. N. Mishra, N. N. Nayak and Swadeenananda Pattanayak, Strong result for real zeros of random polynomials

Sidney Allen Morris and Peter Robert Nickolas, Locally invariant topologies on free groups

Richard Cole Penney, A Fourier transform theorem on nilmanifolds and nil-theta functions

Andrei Shkalikov, Estimates of meromorphic functions and summability theorems

László Székelyhidi, Note on exponential polynomials

William Thomas Watkins, Homeomorphic classification of certain inverse limit spaces with open bonding maps $\ldots \ldots \ldots \ldots \ldots \ldots \ldots \ldots$

David G. Wright, Countable decompositions of $E^{n}$

Takayuki Kawada, Correction to: "Sample functions of Pólya processes" .

Z. A. Chanturia, Errata: "On the absolute convergence of Fourier series of the 